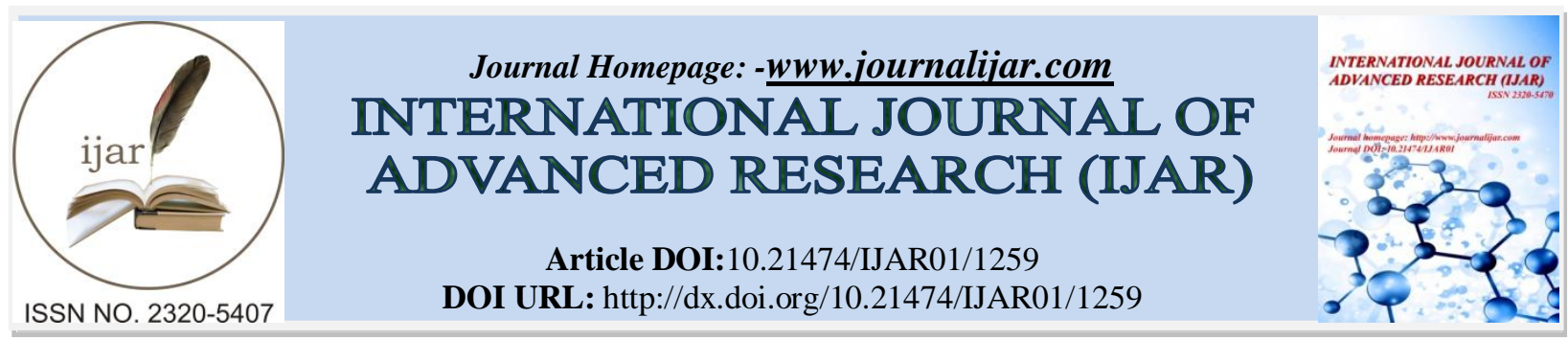

RESEARCH ARTICLE

\title{
ADVANCES IN FUNCTIONAL BRAIN IMAGING: A COMPREHENSIVE SURVEY FOR ENGINEERS AND PHYSICAL SCIENTISTS.
}

*Saman Sarraf ${ }^{1}$ and Jian Sun'2.

1. Department of Electrical and Computer Engineering, McMaster University, Hamilton, Ontario, Canada

2. Department of Engineering Physics, McMaster University, Hamilton, Ontario, Canada

\section{Manuscript Info}

Manuscript History

Received: 19 June 2016

Final Accepted: 25 July 2016

Published: August 2016

Key words:-

Functional brain imaging,

Optical imaging,

fMRI,

\section{Abstract}

Functional brain imaging allows the measure of dynamic functionality in all brain regions. It has highly impacted the clinical and research cognitive neuroscience since its imaging techniques allow the observation of neural activities in the brain simultaneously.

In an earlier era when functional brain mapping was initiated by phrenologists, many scientists questioned the utility of functional mapping given the wealth of structural information. Today, we know that functional brain information can inform and extend structural information, leading to a more complete understanding of brain activity.

This review paper, which is designed be useful to those who have an interest in functional brain imaging, such as engineers or physical scientists, will present a review of non-invasive modalities used in functional brain imaging. This work will also concentrate on established techniques such as functional magnetic resonance imaging (fMRI) as well as emerging techniques such as functional optical imaging.

Copy Right, IJAR, 2016,. All rights reserved.

\section{Brain:-}

Structure and Function:-

The human brain is the most complex organ in the human body with an intricate anatomy which interfaces with almost all other organs through trillions of synapses [1]. The brain includes many complicated regions including the basal ganglia, and cerebellum. The supratentorial cortex is divided into four paired lobes: frontal, parietal, temporal, and occipital [2]. The brain stem, located between the spiral cord and the rest of the brain, controls breathing and sleep. The frontal lobes manage problem solving, judgement and motor function, while the parietal lobes manage body position, hand writing, and sensation. The temporal lobes are responsible for memory and hearing, and the occipital lobes process visual signals [1].

\section{Neurovascular Coupling:-}

A deep understanding of neurovascular coupling is crucial to the interpretation of functional imaging data Figure 1[3]. There is a close coupling between spatiotemporal vascular dilation and neuronal activity, as blood flow is essential for providing oxygen supply and receiving metabolites [4].Histologically, neurons have a close relationship with astrocytes, smooth muscle, pericytes, endothelial cells, erythrocytes, and other glial cells. Neuronal chemo- 
electrical activity is hypothesized to be associated with many metabolic processes, and together this is called neurovascular coupling. Neurovascular coupling includes the following steps: Glucose is metabolized to lactate in astrocytes, and then lactate travels to neurons and is metabolized with oxygen to produce ATP and carbon dioxide. Arteries are responsible for the delivery of oxyhemoglobin (Hbo) to neurons and in the presence of carbon dioxide, oxygen will be released. Consequently, Hbo is converted into deoxygenated hemoglobin (dHbo). Neurotransmitters such as acetylcholine are released by active neurons which cause smooth muscles relaxation in the arterioles, which increases local blood flow and volume [3].

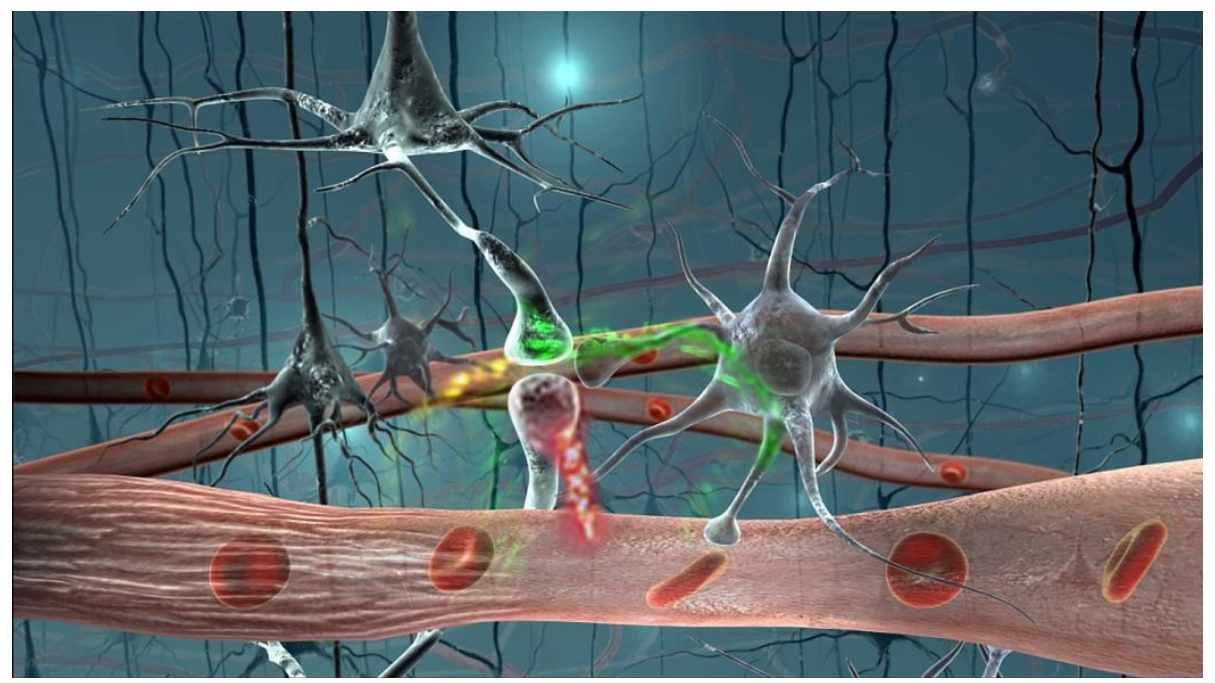

Figure 1:-Neurovascular coupling is associated with a relationship between the local neuronal activity and potential changes in cerebral blood flow. This image subjects to copyright. Permission was obtained from LONI USC. [3]

\section{Temporal and spatial resolution:-}

Cognitive neuroscience is a novel field of study combining cognitive science and cognitive psychology with biology and neuroscience [5]. In 1988, Churchland and Sejnowski proposed a method of brain function study based on temporal and spatial resolution of modalities used in this area. They hypothesized that it could be possible to parse functional brain imaging modalities by temporal and spatial resolution. This basic model has been iteratively improved, and now many novel measurement modalities have been created. Briefly, temporal resolution refers to accurately measuring when an event occurs, and spatial resolution refers accurately measuring where an event occurs [5]. Each modality is categorized by the ability to define brain functionality based on detectable ranges in space and time. Modalities located near the bottom of Figure 2 have better spatial resolution than those at the top while methods situated at the left of figure show better temporal resolution. Therefore, modalities such as Electroencephalography (EEG), Magnetoencephalography (MEG) and Transcranial magnetic stimulation (TMS) demonstrate a good temporal resolution while functional magnetic resonance imaging (MRI) and Positron emission tomography (PET), have sensitive temporal resolution [6]. Functional MRI and functional optical imaging, an emerging modality, are the most utilized imaging techniques for functional brain imaging. In next part, we will look at these modalities and concentrate on fMRI and functional near-infrared spectroscopy (fNIRS).

\section{Imaging Modalities:- Qualitative EEG and MEG:-}

Electroencephalography (EEG) is a technique for the recording of brain activity associated with electrical signals. EEG recording is based on voltage fluctuations resulting from ionic current flows within the neurons. As mentioned before, EEG has a relatively temporal resolution and it is often employed in dual modality fMRI-EEG for functional imaging [7].Electroencephalography is a functional brain imaging technique that records scalp electrical activity by metal or other modern electrodes and conductive media. The electrical signal measured directly from the cortical surface is called electrocorticogram. The depth of activity is simultaneously measured to produce the electrogram. This technique is a completely non-invasive procedure that can be applied repeatedly to subjects such as normal adults, and children with virtually low risk. 
The detail of action potential is out of this paper scope but briefly, activated neurons generate local current flows. Electroencephalogram measures this current flowing during synaptic excitations of the dendrites of many pyramidal neurons in the cerebral cortex. It is fascinating that large populations of active neurons generate sufficient electrical activity that can be recorded on the scalp. In an EEG machine, weak electrical signals recorded by the scalp electrodes are hugely amplified and will be stored and displayed.

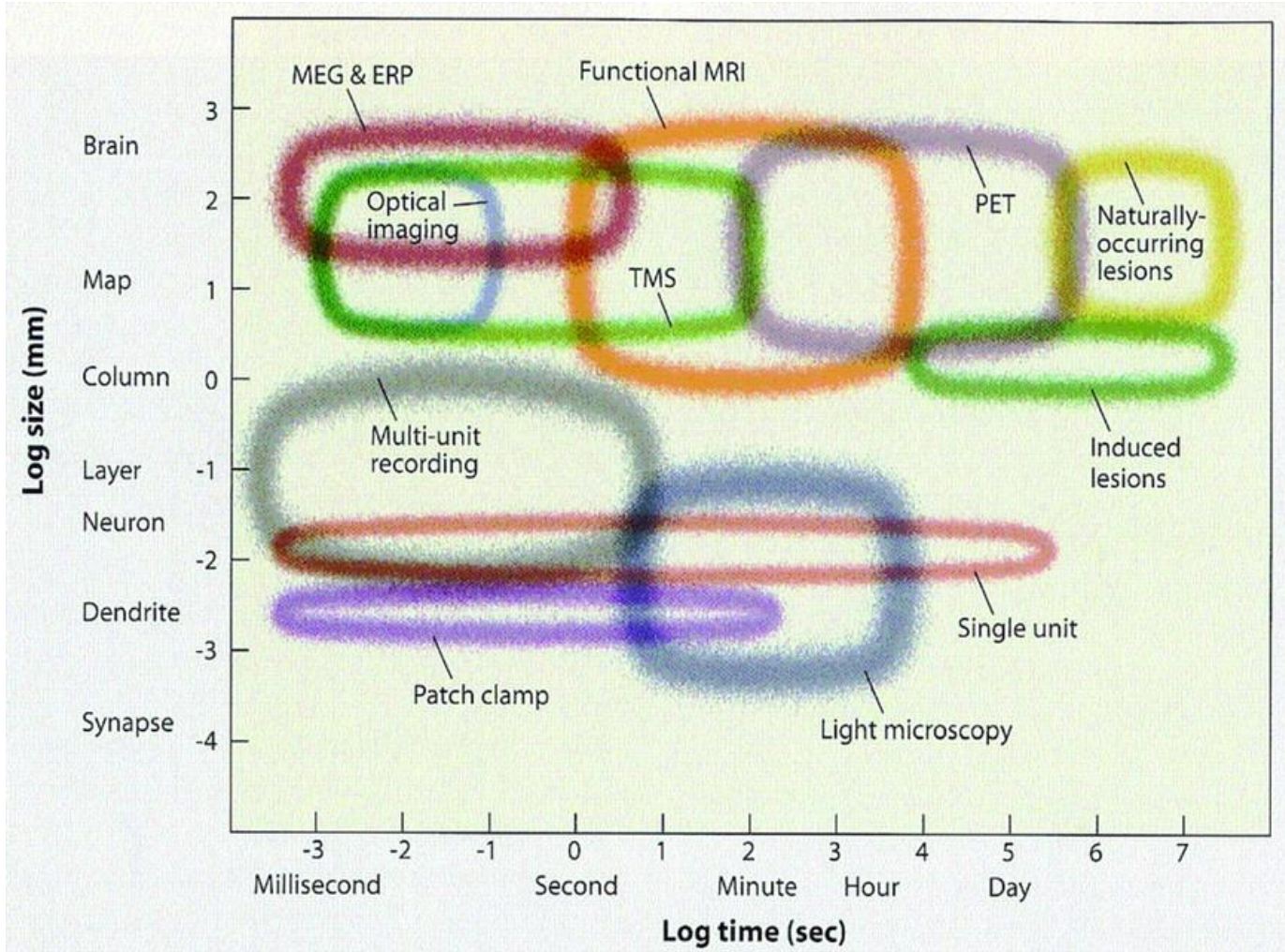

Figure 2:-spatial vs. Temporal Resolution, modalities at left and bottom have better spatial and temporal resolution while the more modalities go to the right and top of the figure, lose the spatial temporal resolution [8].

During EEG recordings, subjects are requested to close their eyes and relax in the resting-state studies. The typical brain wave shapes are mostly sinusoidal and their standard range of the signals measured from peak to peak is between 0.5 and $100 \mu \mathrm{V}$ in amplitude. However, this weak signal is amplified approximately 100 fold in order to generate a sufficient signal for processing and analysis. One of the most popular EEG processing methods is called the Power Spectrum. In Power Spectrum analysis, the contribution of sinusoidal waves with different frequencies can be measured. There are four brain frequencies in EEG: beta $(>13 \mathrm{~Hz})$, alpha $(8-13 \mathrm{~Hz})$, theta $(4-8 \mathrm{~Hz})$, delta (0.5-4 Hz). The predominant waveform in humans is the normal alpha rhythm. Typically, the alpha waveform is observed in the posterior and occipital regions with typical amplitude about $50 \mu \mathrm{V}$ (peak-peak). The International Federation in Electroencephalography and Clinical Neurophysiology adopted standardisation for electrode placement called the10-20 electrode placement system in 1958 (Figure 3).

This system standardized physical placement and designations of electrodes on the scalp. In this standard, the surface of head is divided into proportional distances from prominent skull landmarks (nasion, preauricular points, and inion) to provide adequate coverage of all regions of the brain. Electrodes are then placed at designated spots chosen for their proportional distance between ears and. Electrode placements are labelled according adjacent brain areas: F (frontal), C (central), T (temporal), P (posterior), and O (occipital). The letters are accompanied by odd numbers at the left side of the head and with even numbers on the right side. The convention for left and right side is to assume the point of view of the subject.

As EEG is capable of recording both normal and abnormal electrical activity of the brain, it is a powerful technique used in the field of neurology and clinical neurophysiology [9].Also, this non-invasive technique has been massively 
utilized to monitor brain activity because of low cost and ease of use. An interactive software and hardware platform called the Brain Computer Interface (BCI) has been developed in order to collect and translate EEG signals into meaningful commands to assist disabled people with motor cortex pathologies. In such system, extracting specific features from EEG signals plays a crucial role. One methodology is to categorized signals into: 1 - Signal based methods that directly extract features from preprocessed signals such as Wavelet Packet Decomposition (WPD), Principal Component Analysis (PCA), Haung-Hilbert transformation combining empirical mode decomposition (EMD) algorithm and Hilbert transformation. 2 - Selection based feature extraction methods in which a given system includes three stages such as preprocessing and filtering to remove artifacts, signal segmentation and feature extraction and finally decision algorithms. 3 - Feature extraction based on applications in which the algorithm aims to extract a specific waveform from EEG signal. For example the alpha wave is extracted to design a home care system to monitor mentally disorder patients during treatment[10].
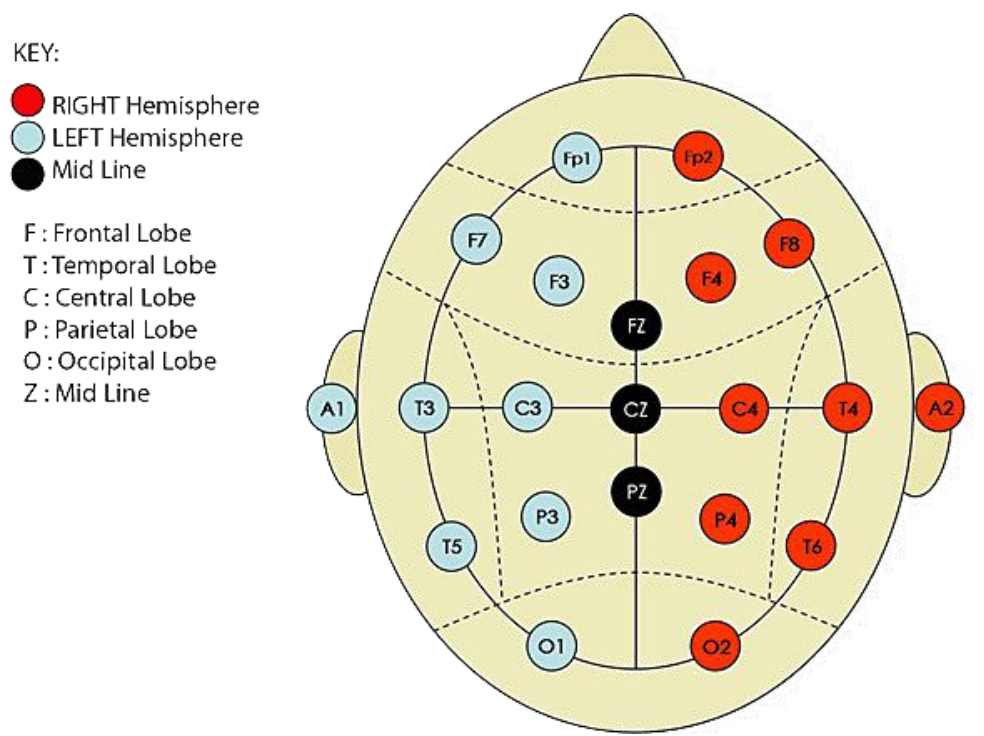

Figure 3:-International 10-20 electrode placement system describes the location of EEG scalp electrodes [11].

Magnetoencephalography or so called MEG (Figure 4) is a non-invasive neuroimaging method to study neuronal activity in the human brain. The temporal resolution is better than $1 \mathrm{~ms}$, while the spatial resolution is 2 or $3 \mathrm{~mm}$ for sources in the cerebral cortex [12]. The electrical currents which are naturally happening in the brain produce magnetic fields which are recorded by MEG technique. Application of MEG includes basic research into cognitive brain processes [13].

During MEG data acquisition, the weak magnetic fields (around $10 \mathrm{fT}-1 \mathrm{pT}$ ) which are produced by electric currents flowing in neurons are measured with a multichannel system using superconducting quantum interference device (SQUID) gradiometers. The different areas in the cerebral cortex of the human brain which are active or stimulated are extracted from the magnetic field distribution.

By using MEG, different functional working human brain activities can be investigated, including spontaneous activity and signal processing following external stimuli. Also, in clinical purposes, MEG can help to determine the locations of epileptic foci. MEG is a famous example of an inverse problem. A forward problem exists when observations are predicted from a model by assuming given parameters. However, in the reverse or inverse situation, observations are used to estimate the model parameters. In MEG, the estimation of the neural sources producing the electromagnetic signals obtained outside the brain is an inverse problem in which the unknown parameters are the source locations and their amplitude as a function of time/frequency [14]. MEG currently has two approved indications in the United States, one is for pre-operative brain mapping and the other is for use in epilepsy surgery [15].

A comparison between EEG and MEG reveals differences between these modalities. In EEG signals are collected by the electrodes placed on the scalp but MEG is performed using a dewar containing multiple sensor coils that are detached from subject's head. MEG primarily measures the magnetic fields induced by intracellular currents while 
EEG is sensitive to electrical fields generated by extracellular currents. To detect an epileptic spike, in MEG, 3-4 $\mathrm{cm}^{2}$ of synchronized cortical epileptic activity is required, whereas at least $6-20 \mathrm{~cm}^{2}$ needed in scalp EEG spike detection. Recently, researchers compared the signal-to-noise ratios (SNRs) of hypothetical sources in different areas of the brain and they showed an advantage for MEG over EEG in the study of neocortical epilepsies. Also, they indicated that the SNRs were comparable in the temporal lobe. MEG data can be analyzed by source modeling techniques to provide 3D localization [15].

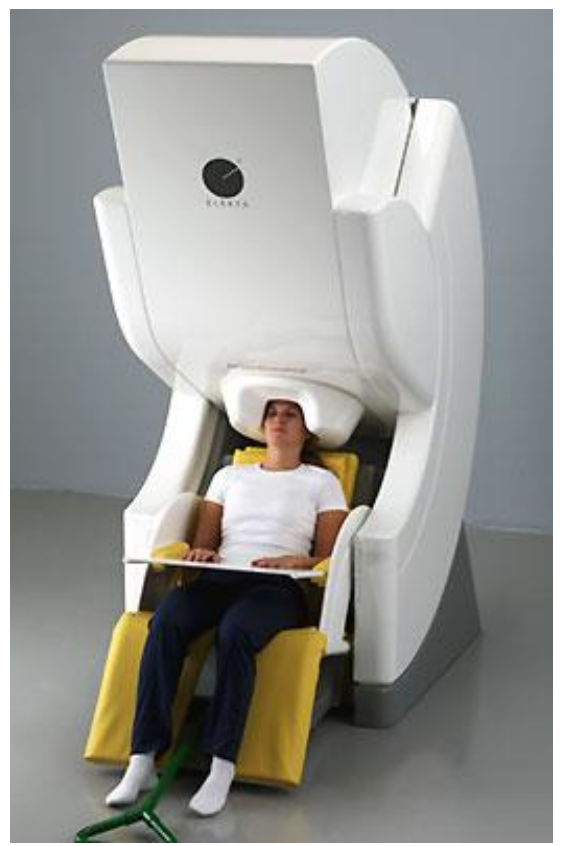

Figure 4:-Magnetoencephalography - Neuromag TRIUX / Elekta . The Neuromag TRIUX agnetoencephalography system is a remarkable diagnostic device for functional brain mapping which is used for the analysis and localization of functional areas of the brain in real time through non-invasive measurement of the magnetic fields produced by the brain

\section{Positron emission tomography (PET):-}

Positron emission tomography (PET) is a nuclear medicine imaging technique which produces 2-D and 3-D images of brain functionality that is widely used for the diagnosis and surveillance of many diseases, including cancer. Physical principles of PET imaging include several steps which begin with choosing the molecule of interest. This molecule has to be labelled radioactively and the product of this step is called tracer production. In this imaging modality, positron-emitting isotope labeled biomolecules are required which are produced before performing image acquisition. One the most commonly utilized radioisotopes is "Fluorine-18" which is one of the several isotopes of fluorine that is often used in radiolabeling of biomolecules because of its positron emitting characteristic and favorable half-life of $109.8 \mathrm{~min}$. The biologically active molecule most frequently used for Positron Emission Tomography is 2-deoxy -2-18F-fluoro- $\beta$-D-glucose (18F-FDG) (Figure 5) which as an analogue of glucose has utility in functional brain imaging and oncologic evaluations. Regional glucose metabolism and accumulation is tracked by the concentrations of tracer accumulation in PET images which acts as a proxy for the metabolic activity of tissues[16].<smiles>O=c1[nH]c(=O)c(=O)[nH]cc1[18F]</smiles>

A

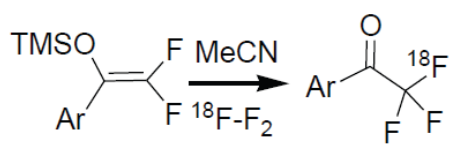

B<smiles>NC(Cc1cc(O)c(O)cc1[18F])C(=O)O</smiles>

C

Figure 5:-Electrophilic fluorination; Syntheses of 18F-5-fluorouracil (A), 18F-a-trifluoromethyl ketones (B), and 18Ffluorodopa (C). [16] 
PET imaging is based on detecting pairs of gamma rays which are indirectly emitted by the positron-emitting radionuclide (tracer) - which will play the role of an active molecule in the body. When sufficient amounts of the labelled molecule are administered into the body, the patients carrying the radioactive trace are placed into the PET scanner. The basic of image acquisition is similar to computed-tomography techniques. The radiation caused by the nuclear annihilation step is the output. Next, the data undergoes image reconstruction. The final goal of PET imaging is to measure the distribution of the tracer. Images obtained by PET imaging will be filtered by cut-off values which help radiologists interpret scans[17][18]. Applications of PET include neuroimaging, oncology, cardiology, musculoskeletal evaluations and small animal imaging. Within neurology, PET is used clinically utilized for the evaluation of movement disorders, epilepsy, brain tumors, dementia, stroke and neuronal plasticity and neuropharmacology [19]. The main clinical application of PET in epilepsy is to localize epileptogenic foci in potential surgical candidates with partial seizures (Figure 6) and to corroborate findings from other investigational modalities such as EEG. In partial seizures, the glucose metabolism and cerebral blood flow in the region of the epileptogenic focus increases during the ictal period. The glucose metabolism remains elevated in this region after the seizure for up to 48 hours before gradually returns to baseline. A decrease in glucose metabolism and blood flow is seen at the epileptogenic focus on interictal PET. It is crucial to combine PET results with concomitant scalp EEG recordings to correlating the PET findings with the clinical data of the patient [19].

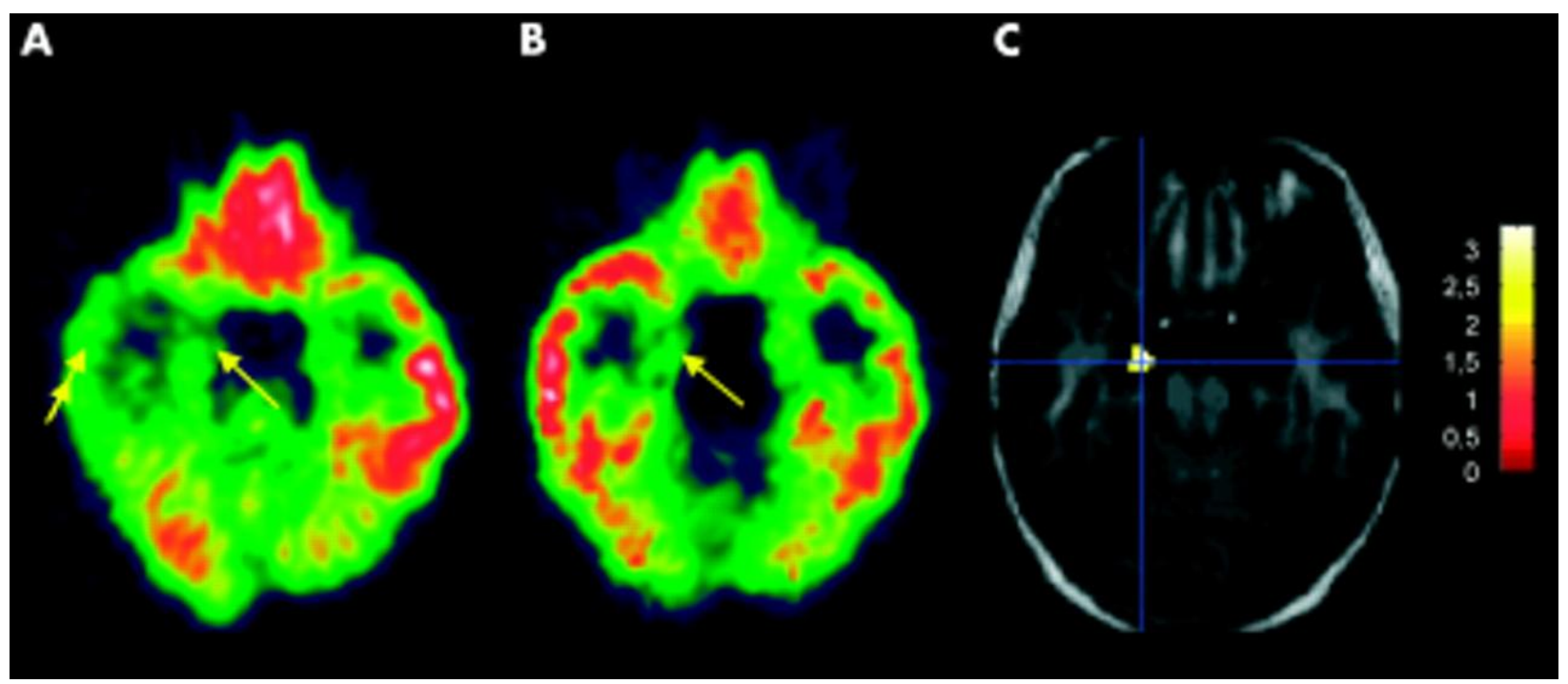

Figure 6:- Comparison of 18FDG PET (A) and 11C-FMZ PET (B) in a 28 year-old patient with complex partial seizures, interictal left temporal epileptiform discharges and left temporal seizure onset on video telemetry. (A) 18FDG PET shows extensive hypometabolism in the left temporal lobe, affecting both the medial temporal cortex (arrow) as well as the inferior and lateral (double arrow) temporal neocortex. (B) 11C-FMZ-PET shows a circumscribed decrease of 11C-FMZ volume-of-distribution (Vd) in the left medial temporal lobe (arrow). (C) Statistical analysis with statistical parametric mapping localised a significant decrease of FMZ-Vd in the left anterior hippocampus, compared with 21 controls (Images courtesy of A Hammers.) [19]

\section{Single-Photon Emission Computed Tomography (SPECT):-}

Brain SPECT is a powerful clinical and research tool for the management and diagnosis of neurologic diseases [20]. SPECT is a nuclear medicine imaging modality which provides information on cerebral function and similarly to other nuclear medicine techniques uses a radiopharmaceutical tracer [21]. Poor image quality lessens its clinical utility. In place of detecting two gamma-photons as in PET, there is only one produced photon in SPECT. Therefore, the image quality of PET is much higher than SPECT. This modality is utilized in functional brain imaging and also in myocardial perfusion imaging. The radioisotopes typically used in SPECT include iodine-123, technetium-99m, xenon-133, thallium-201, and fluorine-18. The radioactive forms of these natural elements will pass safely through the human body and be detected by the scanner. Table 1 compares SPECT and PET advantages and disadvantages. One clinical application for SPECT is for preoperative evaluation of medically uncontrolled seizures. The test can be performed between seizures (interictal) or during a seizure (ictal) to determine blood flow to areas where the seizures originate. The comparison between PET and SPECT shown in (Table 1) reveals some advantages of using PET. 
Table 1:-Comparison between SPECT and PET shows pros and cons of each modality such as the image resolution which is higher in PET.

\begin{tabular}{|l|l|}
\hline PET & SPECT \\
\hline Emits positrons & Emits gamma radiations \\
\hline Higher resolution & Lower resolution \\
\hline Costlier scanner & Less capital intensive scanner \\
\hline Limited half-life of radiopharmaceuticals & Longer lived radioisotopes \\
\hline Higher Sensitivity & Lower Sensitivity \\
\hline Higher Signal to Noise Ratio (SNR) & Lower SNR \\
\hline
\end{tabular}

\section{Functional Magnetic Resonance (fMRI):- \\ Introduction:-}

Functional Magnetic Resonance Imaging has affected cognitive neuroscience over the past decade. This imaging modality is a technique which measures brain activity by detecting the associated changes in blood flow. By taking advantage of the coupling between neuronal activity and haemodynamic response (as mentioned earlier), fMRI provides information or complementary data to more invasive techniques in order to explore the functionality and dysfunctionality of the human or animal brains. This basis for this technique, which is similar to MRI, uses the detection of magnetization differences between oxygen-rich and oxygen-poor blood.

The foundation for magnetic resonance imaging dates back to 1946. Felix Bloch (1905-1983) who was at Stanford University studying liquids, and Edward Purcell (1912-1997) who was working on solids at Harvard University, both made important contributions. Although they could have won Nobel Prizes for these discoveries, it was not until 1973 that successful nuclear magnetic resonance (NMR) was used to produce images. Finally, in the 1990s the discovery that changes in blood oxygenation level could be detected with the use of MRI opened a window on a new generation of functional brain imaging techniques [22].

\section{MR Signal Generation and Formation:-}

Magnetic resonance signal generation requires the review of several concepts. First, atomic nuclei have magnetic momentum as well as angular momentum which are called nuclear spin, and this plays an important role. "Two axes are important here which are longitudinal direction and transverse plane, respectively. Longitudinal direction is the axis around which an atomic nucleus precesses and the transverse plane is the plane on which a nucleus precesses" [22]. Each spin will adopt a low or high energy state (different magnetic potential energies) which can be parallel or antiparallel to the magnetic field. By applying an electro-magnetic pulse oscillating at the resonant frequency (Larmor or precessional frequency) of the spins, the atoms will undergo excitation and the net magnetization vector will change from the longitudinal to the transverse plane [22]. With time (called evolution), the net magnetization changes in the transverse plane generates MR signals. MR signals can be measured in the external coil. The Bloch equation (Equation 1) describes quantitatively the behaviour of magnetization in the presence of a time-varying magnetic field [22][23].

Equation 1:- The Bloch Equation

$$
\frac{d M}{d t}=\gamma M \times B+\frac{1}{T_{1}}\left(M_{0}-M_{z}\right)-\frac{1}{T_{2}}\left(M_{x}+M_{y}\right)
$$

The Bloch Equation, a quantitative description of magnetic resonance where $M$ is net magnetization, $\gamma$ is gyromagnetic ration, $B$ is magnetic field, $\mathbf{T} 1$ is the time constant of longitudinal relaxation process and $\mathrm{T} 2$ is the time constant of transverse relaxation.

As described by the Bloch equation, the net magnetization of a spin can be divided into separate components along the spatial coordinates $(\mathrm{x}, \mathrm{y}, \mathrm{z})$. "The longitudinal magnetization is defined as $\mathrm{M}_{\mathrm{z}}$ and the transverse magnetization are defined as $\mathrm{M}_{\mathrm{xy}}$ " [22]. $\mathrm{T}_{1}$ contrast is related to the time of recovery of the longitudinal magnetization (excitation) and $\mathrm{T}_{2}$ contrast is associated with the decay of transverse magnetization [22]. Briefly, there are several steps to achieve a 3-D MR image. First, as mentioned before, longitudinal magnetization happens following the volume excitation. The next step is transverse magnetization, where 3-D spatial encoding is performed in order to acquire a MR signal in K-space, resulting in a 3-D MR image [22][24]. 
$\mathrm{K}$-space is a 3D Fourier transformation of the acquired data. Its complex values are sampled during an MR measurement, in a premeditated scheme controlled by a pulse sequence, i.e. an accurately timed sequence of radiofrequency and gradient pulses. In practice, k-space often refers to the temporary image space, usually a matrix, in which data from digitized MR signals are stored during data acquisition. When k-space is full (at the end of the scan) the data are mathematically processed to produce a final image. Thus k-space holds raw data before reconstruction. As described, image acquisition in MRI is using the K-space idea affected by a Fourier transform of the image space.

\section{MR Contrast Mechanisms:-}

Generally, the mechanisms of MR contrasts are divided to four main categories. 1) Static Contrast which are sensitive to the type, number and relaxation properties of atomic nuclei. This category includes, $\mathrm{T}_{1}$-wieghted, $\mathrm{T}_{2}-$ wieghted, $\mathrm{T}_{2}^{*}$ weighted and proton density contrast (Figure 7). The main application of static contrast is to determine anatomic information of the brain in fMRI. 2) Motion Contrasts are sensitive to the movement of spins in the space. This group which includes diffusion and perfusion-weighted, will define dynamic properties of the proton in the brain such as blood flow through MR angiography. 3) Endogenous contrast is related to an internal feature of biological tissue in the brain while 4) Exogenous contrast needs an external agent injected to the body playing the role of contrast. For instance, BOLD fMRI is an endogenous contrast because it uses the blood flow properties, but injected Gadolinium $(\mathrm{Gd})$ is an example of exogenous contrast [22].
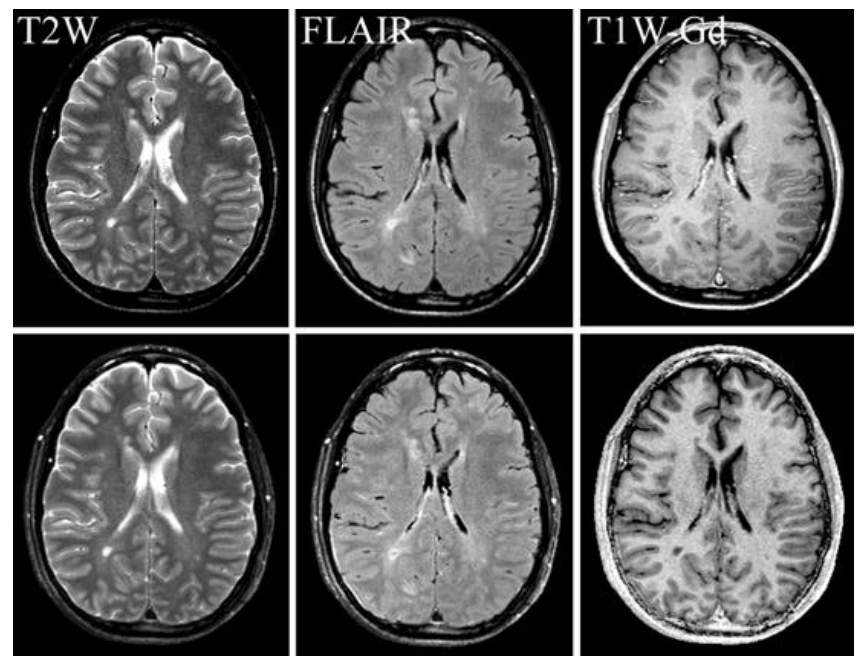

Figure 7:-Comparison of conventional and Synthetic Contrast MRI. T2-weighted, T2-weighted FLAIR and T1weighted post-Gd contrast of the same slice. The first row was acquired conventionally, the second row has been synthesized [25]

\section{Image contrast:-}

The number of spins in a given volume will determine a MR signal resulting in spatial contrast. The equation below shows that image contrast is affected by other parameters. In fact, image contrast (Equation 2) will be determined by $\mathrm{T}_{\mathrm{R}}$ and $\mathrm{T}_{\mathrm{E}}$. For instance choosing a quite small $\mathrm{T}_{\mathrm{E}}$ will eliminate $\mathrm{T}_{2}$-weighted images [3]. This short description might be of some mathematical interest. Image contrast equation where $\rho$ is the density of nuclear spins, $T_{R}$ is repetition time, $T_{E}$ is echo time, and $\alpha$ is flip angle. This equation shows that $T_{1}$ will be maximized by $T_{R}$ and by using a flip angle equal or greater than the Ernest angle.

Equation 2:-Image Contrast Equation.

$$
S \sim \rho \frac{1-\exp \left(-\frac{T_{R}}{T_{1}}\right)}{1-\cos \alpha \exp \left(-\frac{T_{R}}{T_{1}}\right)} \sin \alpha \exp \left(\frac{-T_{E}}{T_{2}}\right)
$$

\section{BOLD fMRI:-}

Blood Oxygenation Level Dependent fMRI (BOLD fMRI) is a technique which indirectly detects neural activity, based on magnetic properties of hemoglobin. Because of molecular differences, when Hbo and dHbo are placed in a 
magnetic field, dHbo will act as a contrast agent. In BOLD fMRI, the change in the concentration of dHbo is dynamically monitored. Changes and correlations between tasks (stimulation) and concentration of Hbo will be considered. A widely accepted assumption is that as neuronal activity and blood flow are directly correlated.

This results in local concentration alterations of dHbo which produces the BOLD fMRI signals. This measurement is iterated until the completion of the scan. Importantly, BOLD fMRI signals are relevant to three physiological concepts: Glucose and $\mathrm{O} 2$ consumption, blood flow and volume, and concentration of dHbo, respectively [26]. Figure 8draws the hierarchy of BOLD signal generation following neuronal activation. In other words, the hydrogen atoms which are plentiful in the water molecules of the brain are the source of signals for blood oxygen level dependent fMRI. In the MRI machine and the presence of a magnetic field, the hydrogen atoms absorb energy with a frequency around $64 \mathrm{MHz}$ for a standard - clinical 1.5-Tesla MRI. After this excitation step, energy is emitted until they gradually return to their equilibrium state. The MRI scanner measures the sum total of the emitted radiofrequency energy. Various factors play roles in decaying RF signals over time such as inhomogeneities in the magnetic field.

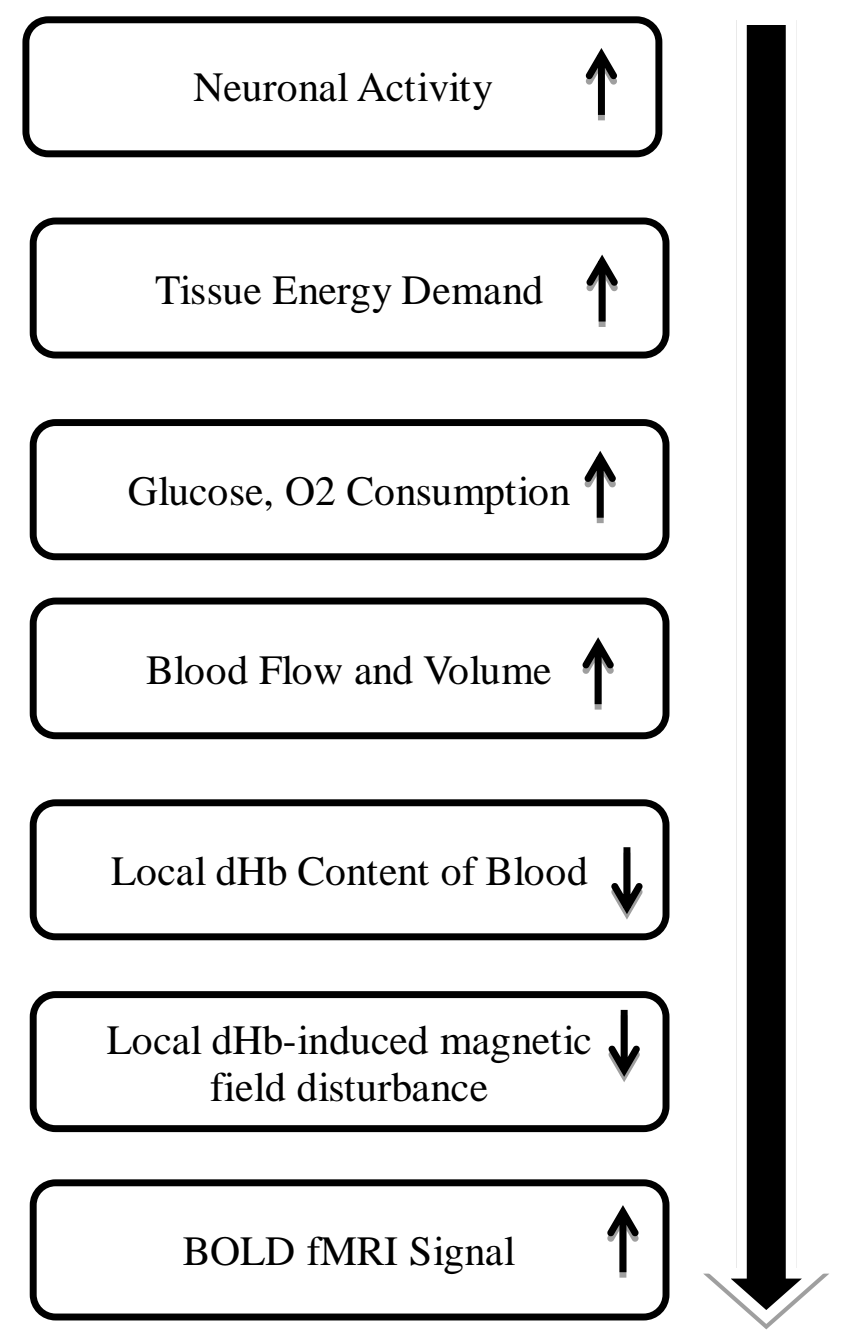

Figure 8:-In Functional MRI, the brain activity through changes in blood flow is measured. The process of producing BOLD signals is shown in the above figure. BOLD fMRI techniques are to measure alterations in the inhomogeneity of the magnetic field, within each small volume of tissue resulting from changes in blood oxygenation. Deoxy- and oxyhaemoglobin have different magnetic properties while deoxyhaemoglobin is paramagnetic inducing an inhomogeneity into the magnetic field, whereas oxyhaemoglobin is slightly diamagnetic. Therefore, once the concentration of deoxyhaemoglobin increases, it would cause a decrease in signal intensity, followed by a decrease in deoxyhaemoglobin causing an increase in BOLD signal intensity. 


\section{Pre-processing and Statistical Analysis of fMRI data:-}

Preprocessing is used to improve experimental analysis. The quality and clarity of the generated images, which is critical for accurate medical diagnosis, is assured by this step. Preprocessing of fMRI data are based on four principal phases. First, recording of fMRI signal is done followed by an image reconstruction. Then the online quality assurance is applied. Quality assurance, called Q.A, is a set of procedures defining errors in fMRI data, to avoid continuing the process with those errors. The second phase will be head motion correction, as well as slice timing correction. Each slice is acquired at a different time point within the TR, and the timing difference are especially problematic for an interleave sequence, in which spatially consecutive slices are not achieved successively. Slice timing correction accounts for resolving this issue. This phase is a recursive procedure and it is repeated to the best possible correction. In the third phase, distortion correction, functional and structural coregistration normalization and spatial/temporal smoothing (image smoothing) are respectively performed. Finally, the online quality assurance is repeated again and fMRI image are provided [22]. In Figure 9, the block diagram of fMRI preprocessing pipeline is illustrated. Several research groups across the world have been developing and providing fMRI preprocessing pipelines[3][27][28]. Figure 9 (bottom image) shows LONI pipeline developed by Laboratory of Neuro Imaging - University of Southern California.

Since the fMRI provides vast information which can be challenging to interpret, a variety of statistical approaches are used in the analysis. The basic statistical tests such as t-test or K-S test, as well as frequency domain analysis methods such as Fourier or Wavelet analysis are applied on data generated by fMRI. Furthermore, pattern classification methods such as artificial neural networks or graph theory may be useful to study fMRI data[22][23].

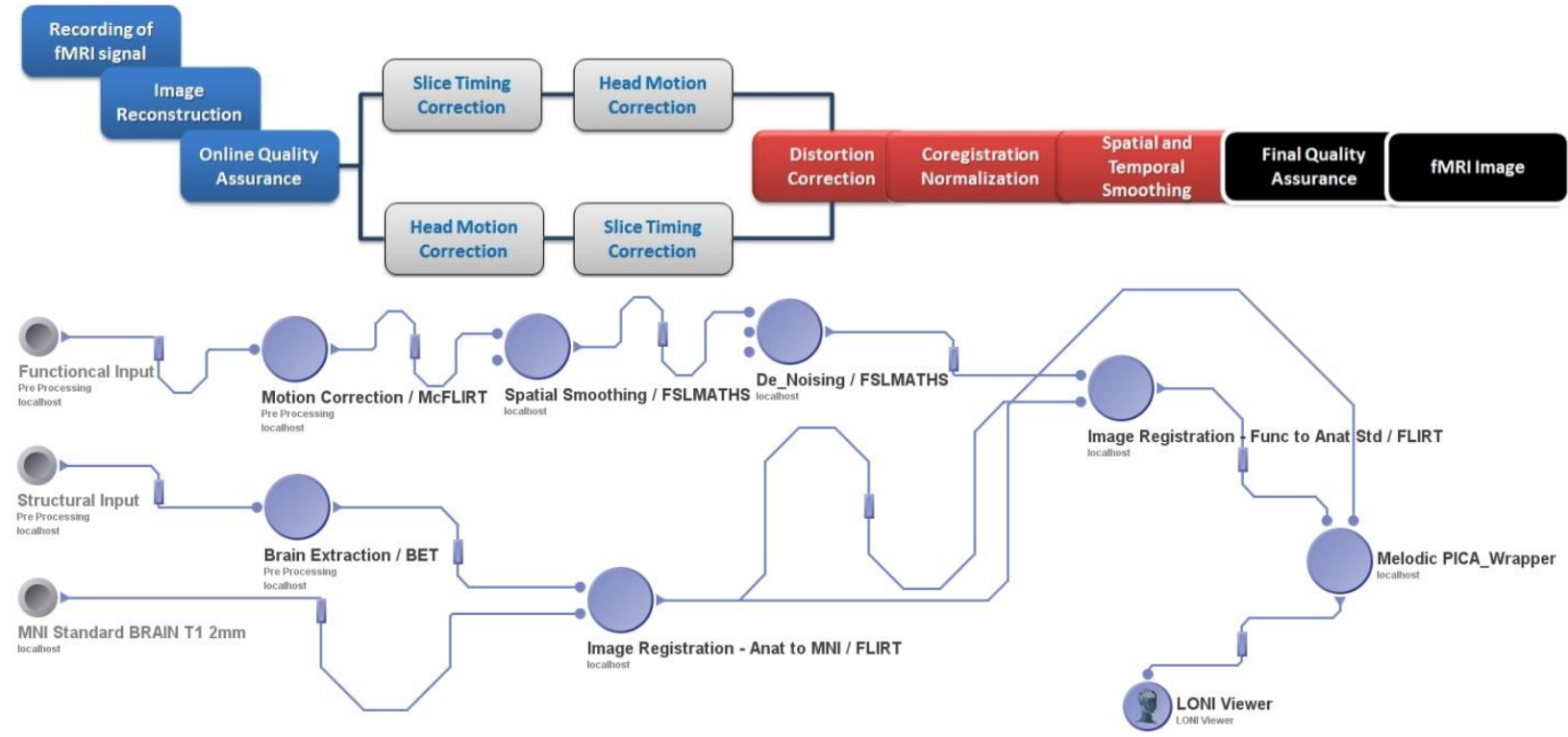

Figure 9:-Framework above shows the different phases of pre-processing of fMRI data. LONI has developed an fMRI preprocessing pipeline using free FMRIBsoftware libraries.

\section{Resting-state fMRI:-}

A novel technique used in fMRI is called resting-state where fMRI data acquisition is performed while no requested task is doneby subjects. This increases the comfort of the procedure and provides baseline measurements which can simplify data interpretation by allowing specific extraction of non-task activated brain regions. [29][30].

Low frequency oscillations ( $\sim 0.01-0.1 \mathrm{HZ})$ are the most commonly observed phenomenon during resting-state BOLD signals are from the of fMRI time-series. However, the interpretation of these low frequency signals is still unclear. Many researchers have been conducting various studies to understand why in certain frequencies, some regions in the brain show higher correlation in the BOLD signals at rest. 
Resting-state networks, like the default mode network as shown in

Figure 10 and the lateralized attentional parietal-frontal networks (as well as the primary motor and visual networks) often consist of anatomically separated cortical regions while those brain regions are able to remain functionally connected. If resting-state oscillations truly reflect ongoing neuronal activity and communication between brain regions, it is postulated that direct structural connections must exist to support this ongoing communication. As we know, structural connections in the brain are white matter tracts including millions of long distance axons. Some researchers believes in this hypothesis that white matter tracts transmit signals that contain the information and enable transport of large amount of functional data between spatially separated regions. Hence, the correlation of resting-state fMRI signals between structurally separated regions are justified by existence of structural paths called tracts in the brain which are transmitting the neuronal communication [31].
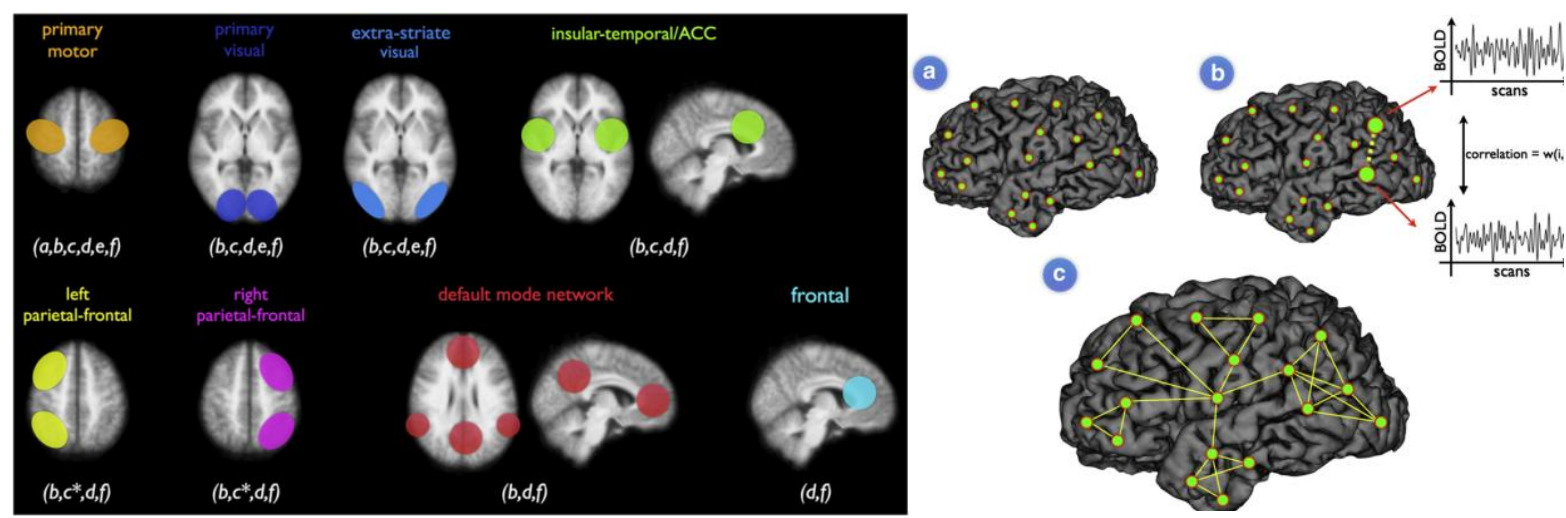

Figure 10:-Resting-state networks. A number of group resting-state studies have consistently reported the formation of functionally. (Heuvel et al., 2010)

For studying resting-state fMRI data, several methods can be applied. The most used approach is seed voxel pictured in Figure 11. The premise is that a voxel is considered in the MR image which is anatomically known. As each voxel represents a signal, all signals which may have a high correlation to the seed voxel are identified. After finding all correlated signals, they consider those in a cluster and a network in the brain is achieved [29][30] .

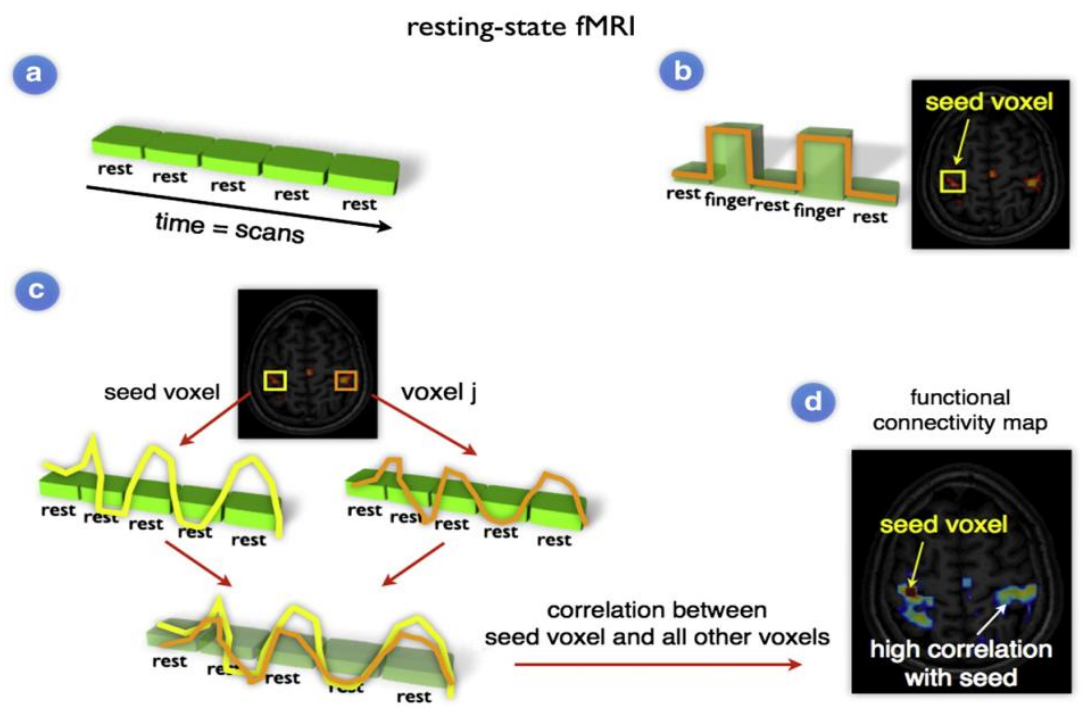

Figure 11:-Resting-state fMRI studies are focused on measuring the correlation between spontaneous activation patterns of brain regions [31].

Another approach which is useful to study resting-state fMRI data is called graph analysis. A graph including clustering-coefficient and connectivity degree, etc. is formed and with the help of this graph, the networks in the 
brain can be identified. According to [31] several networks such as primary motor, primary visual, extra-striate visual and insular-temporal/ACC have been identified by using the above mentioned approaches. The unique feature of graph-theoretical analysis, compared with the more traditional univariate neuroimaging approaches, is that it can directly test the differences in topological parameters of the brain network such as small-worldness, modularity, highly connected regions (hubs), and regional network parameters. [32]. Hosseini et al. has developed a GAT: Graph Analysis Toolbox available at: https://www.nitrc.org/projects/gat/.

Recently, [33] showed a new application of graph theory (Figure 12) in aging studied by measuring the between and within network interactions for DAN, dorsal attention network; DMN, default mode network (BW Toolbox available at: https://github.com/samansarraf/BW_Toolbox).

Furthermore, various research groups have developed medical image analysis algorithms to extract brain networks and also have used multivariate techniques to analyze fMRI resting state and task data [34][35]. To summarize, "Functional connections of resting-state networks is related to structural white matter connections suggesting a structural core of functional connectivity networks in the brain" [31].
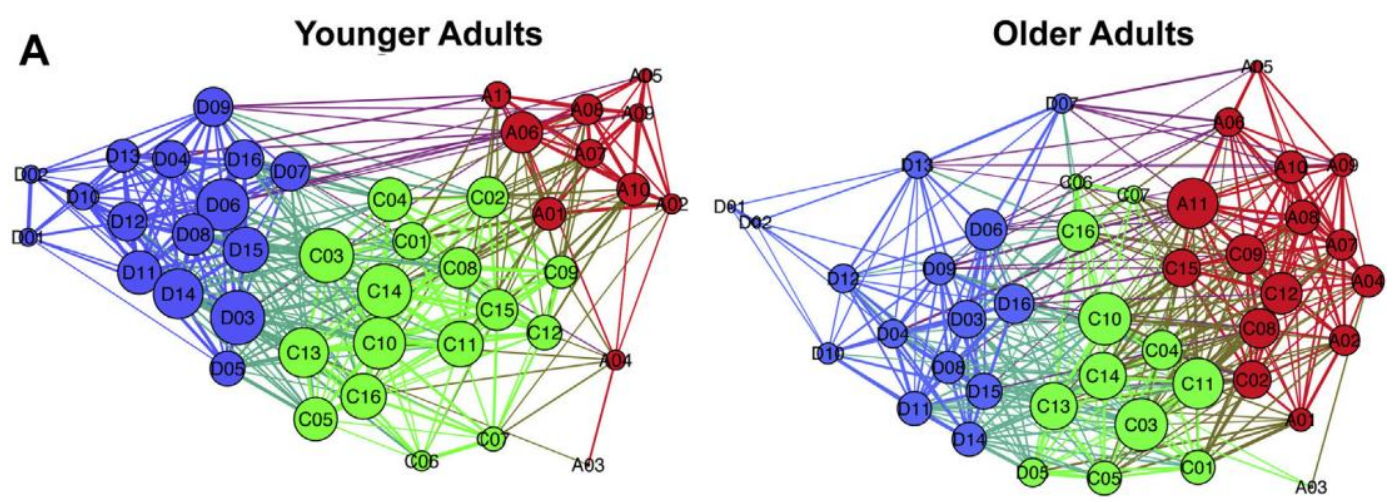

B
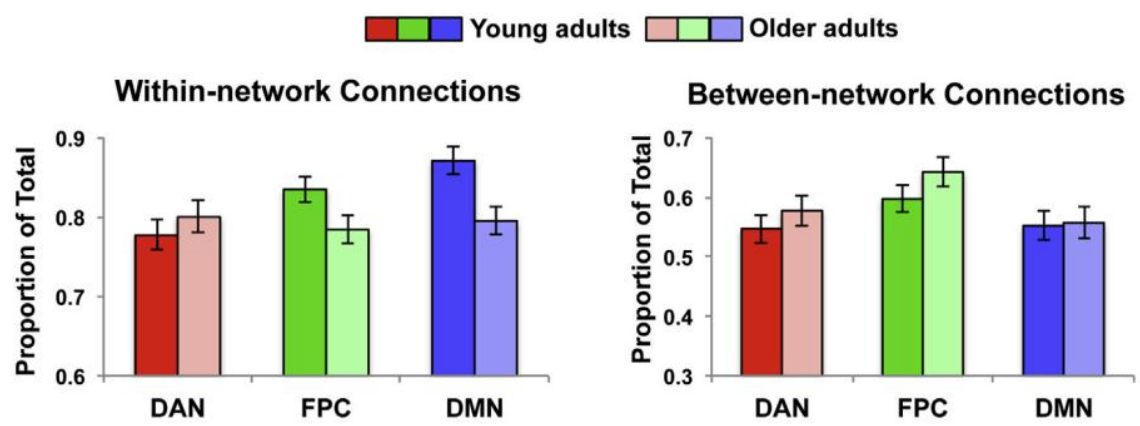

Figure 12:-The graphs (A) and within- and between-network connections (B) for young and older adults are shown for rest. The DAN is represented in red, FPC in green, and DMN in blue. The FPC lies between the other 2 networks in both groups, although some nodes have been assigned to non-canonical networks in the older group. Each metric (in B) was calculated as the proportion of connections within or between networks (out of the total possible) for each node and then averaged across nodes for each network. Reassigned nodes in older adults were considered to be part of the network as they appear in (A) (e.g., nodes C02, C08, C09, C12 and C15 were included in the DAN for the calculations seen in [B]). Abbreviations: DAN, dorsal attention network; DMN, default mode network [33].

\section{Functional Brain Imaging and Multiple Modalities:-}

Currently, single modality medical imaging is used for diagnostic purposes, but an emerging paradigm is that simultaneous multimodality imaging could capture considerably more information which could improve diagnostic accuracy. The current paradigm of sequentially performing a series of diagnostic scans with different modalities in different places requires the information to be integrated for a final diagnosis to be rendered. Thus, the idea of dual modality (hybrid modality) could be realized, especially when a combination of structural and functional imaging data were needed to lead to a better interpretation [36]. 
Several practical problems exist which limit the use of multimodality imaging system currently. The major problem is the difference between temporal resolutions of modalities which has to be combined. Other software limitations such as image fusion and hardware restrictions are still a challenge in this area. Hopefully, additional advances will be made in dual modality imaging allowing for great commercialization in the future.

Table 2 illustrates several imaging (non-imaging) systems used in hybrid modalities [37].

Table 2:-Technical feasibility of simultaneous multimodal imaging [37]

\begin{tabular}{|c|c|c|}
\hline Combination & References & Comments \\
\hline \multirow[t]{5}{*}{ EEG-MRI } & Ives et al. (1993); Busch et al. (1995) & \multirow{5}{*}{$\begin{array}{l}\text { Although the feasibility of this combination was } \\
\text { shown a few years back, broad usage started with } \\
\text { further development in equipment, artefact } \\
\text { elimination and analysis }\end{array}$} \\
\hline & Bonmassar et al. (1999,2001); & \\
\hline & Allen et al. (1998,2000); & \\
\hline & Goldman et al. (2000); Krakow et al. (2000); & \\
\hline & Lemieux et al. $(1997,2001)$ & \\
\hline \multirow[t]{6}{*}{ NIRS-MRI } & Kleinschmidt et al. (1996); & \\
\hline & Kida et al (1996); & \\
\hline & Punwani et al (1998); & \\
\hline & Toronov et al. (2001); & \\
\hline & Mehagnoul-Schipper et al. (2002); & \\
\hline & Strangman et al. (2002) & \\
\hline TES-MRI & Brandt et al. (1996) & \\
\hline MRI-MEG & Zotev et al. (2008) & \\
\hline fTCD-MRI & & $\begin{array}{l}\text { Probably feasible, since combined ultrasound } \\
\text { and MRI systems have been demonstrated } \\
\text { (McDonnald et al. 2003) }\end{array}$ \\
\hline PET-NIRS & Villringer et al. (1997) & \\
\hline PET-fTCD & Sabri et al. (2003) & \\
\hline \multirow[t]{4}{*}{ PET-EEG } & Buschsbaum et al. (1984); & \\
\hline & Sadato et al. (1998); & \\
\hline & Barrington et al. (1998); & \\
\hline & Gamma et al. (2004) & \\
\hline PET-MEG & & $\begin{array}{l}\text { Feasible in principle, but no example of successful } \\
\text { combination was found }\end{array}$ \\
\hline PET-CT & & $\begin{array}{l}\text { Mainly used in clinical oncology (Beyer et al. } \\
\text { 2000) }\end{array}$ \\
\hline \multirow[t]{2}{*}{ PET-MRI } & Catana et al. (2008) & \\
\hline & Judenhofer et al. (2008) & \\
\hline \multirow[t]{2}{*}{ EEG-MEG } & Salustri et al. (1989) & \\
\hline & Buchner et al. (1994) & \\
\hline \multirow[t]{4}{*}{ EEG-NIRS } & Hoshi et al (1994); & \\
\hline & Steinhoff et al. (1996); & \\
\hline & Kirkpatrick et al. (1998); & \\
\hline & Obrig et al. (2002) & \\
\hline MEG-NIRS & Mackert et al. (2004) & \\
\hline NIRS-TCD & Terborg et al. (2003) & \\
\hline
\end{tabular}

\section{Functional Brain Imaging - Optical Approach:- Introduction:-}

Historically, direct optical observation has provided some of the earliest functional observations in brain [38][39]. In the past three decades, advances now allow non-invasive, three dimensional functional optical imaging of the human brain or in vivo in living animal brain tissues. Functional optical brain imaging carries significant importance for understanding the physiological basis of neuron activities, because of its high temporal resolution and high sensitivity that other well-established clinical imaging modalities cannot provide. PET can provide 3-D image using 
fluorodeoxyglucose (FDG) labeled with radioactive isotope. However both spatial and temporal resolution is low. CT is able to provide high resolution image with fast data acquisition, but cannot easily obtain functional information. Functional MRI (fMRI) using the blood oxygen level dependent (BOLD) signal provides excellent spatial resolution. However, the achievable temporal resolution is still limited by its long data acquisition time. As optical imaging does not depend on ionizing radiation, it has substantially lower infrastructure costs, and requires minimum footprint and maintenance.

Functional optical brain imaging can be either invasive or non-invasive. High resolution brain imaging requires direct exposure to laser light, such as multi-photon fluorescent microscopy [40], which has been widely adopted in animal neuron imaging researches. Non-invasive imaging generally refers to functional near-infrared spectroscopy (fNIRS), which detects the hemodynamics in brain tissues. It has provided useful complimentary information to in several clinical domains including the study of cognitive activity [41], Alzheimer's disease [42][43] [44], stroke [45] [46] and birth asphyxia [47][48][49]. The current problem for 3-D optical imaging is scattering, which limits the achievable image quality and the penetrable depth. As a result, most optical brain imaging studies have been limited to examination of brain cortex.

\section{Microscopic Functional Imaging:-}

Microscopic study of neuron networks provides basic yet significant understanding of cellular and molecular activities in the brain. Analyzing the neural circuits in vivo helps develop network topologies that give us fundamental information about their interactions and functionalities. Two-photon microscopy has caught much attention since it was first invented in 1990 [40], a few years after the invention of Ti-sapphire laser in the early [50]. Instead of exciting the fluorophores at visible wavelength, the two photon microscope utilizes femtosecond laser at near infrared wavelength to complete excitation via two photon absorption, which is a third-order nonlinear optical process. As a result, only the fluorophores at the focal point of the beam can be excited. 3-D scanning can be realized by adding galvo mirrors or acoustic optical deflectors (AODs) in the optical assembly. Since the infrared light can penetrate into tissues with lower scattering, and its wavelength is well apart from the fluorescent wavelength, the two photon microscope is able to provide 3-D image with unprecedented spatial resolution. Figure 13 and Figure 14 show the super resolution of fluorescent microscopy and also the schematic structure of the microscope, respectively. Over the past decade, two-photon microscopy has become a well-established method for in vivo optical neuroimaging [38] [51][52][53].
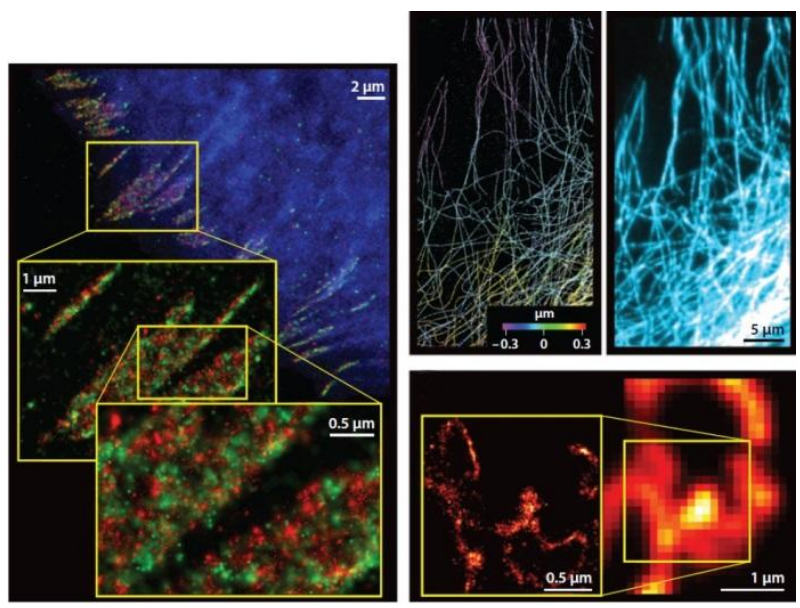

Figure 13:-Super-resolution fluorescent microscopy [54] 


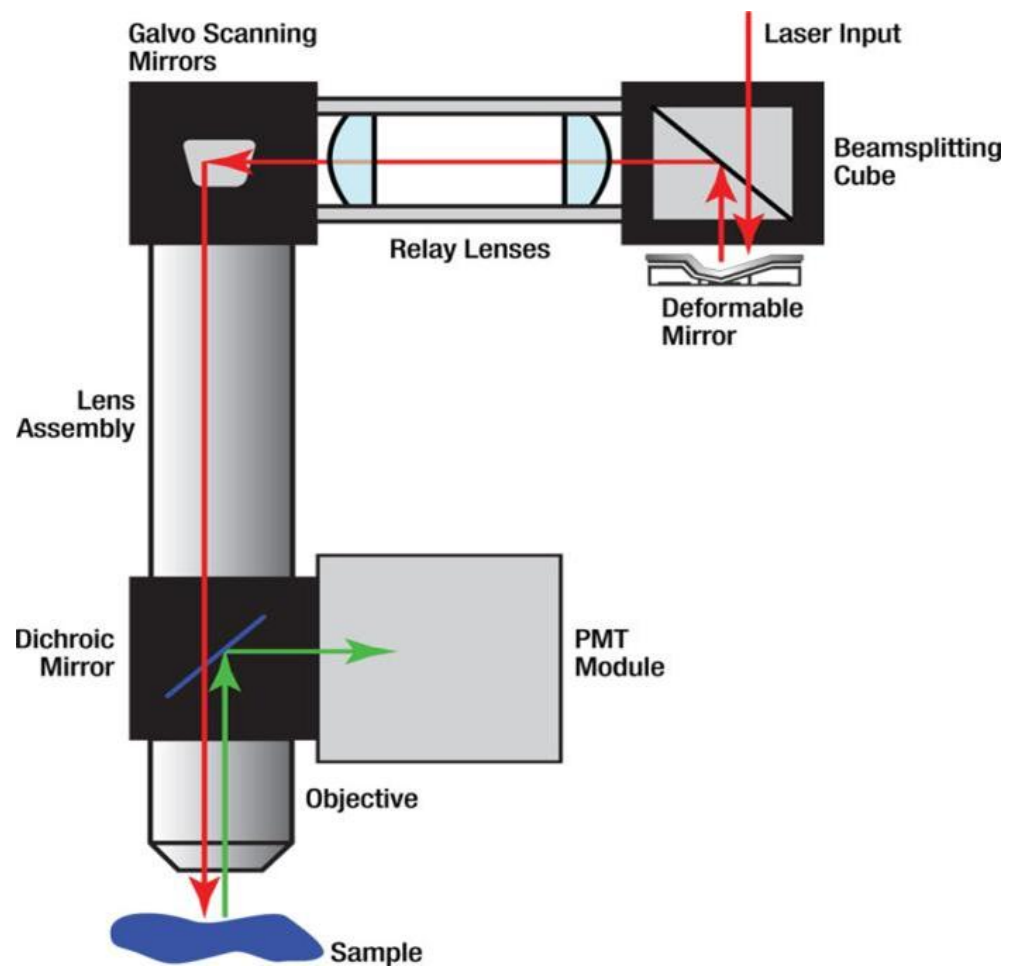

Figure 14:-Schematic diagram of a two photon fluorescent scanning microscope.

Intracellular calcium ion concentration is closely linked to many cellular functions. Functional imaging in vivo can be achieved by use of membrane-permeable calcium sensitive dyes such as Oregon Green 488 BAPTA-1 acetoxymethyl ester [51][55]. This dye is injected via a pipette to the interested area. After diffusing into a cell, it is hydrolyzed by endogenous esterases and stays within the intracellular fraction, while the remaining extracellular dye is gradually washed out. This results in a uniform staining of all cells in the field of view. This method is called the multi-cell bolus loading (MCBL) technique, by which fluorescent observations can be performed in intact cortical neuron cells. An example of two photon microscope image of neuron ensembles is shown in Figure 15.
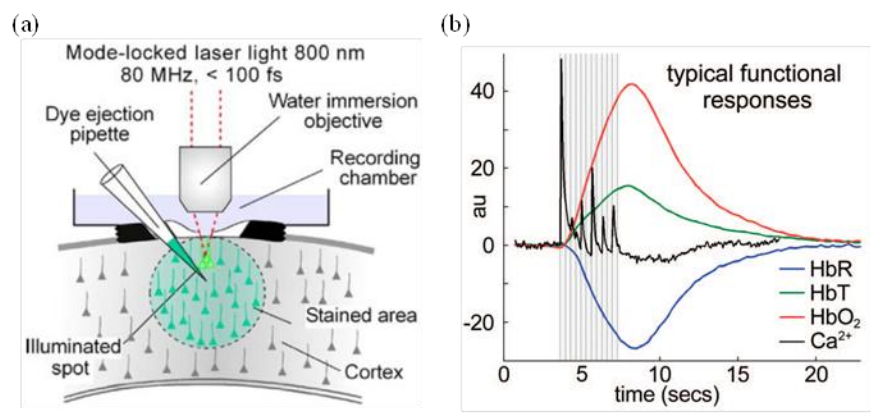

(c)

(d)
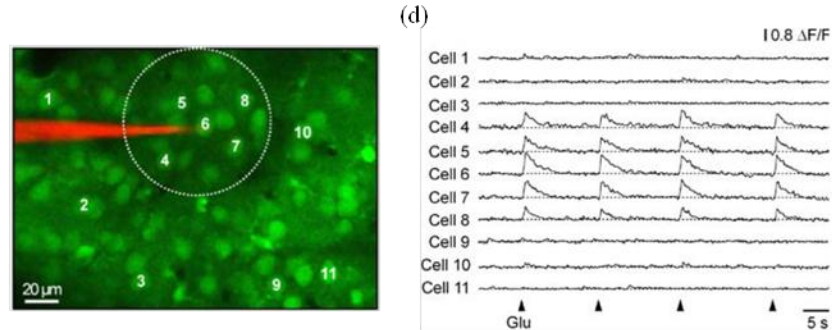

Figure 15:-(a) The MCBL method for neuron cell staining (O. Garaschuk, 2006) (b) Typical Ca2+ and hemodynamic response of a neuron cell after stimulation (E. M. C. Hillman et al., 2007). (C) and (d). In vivo Ca2+ transients in mouse visual cortex, the circle in (c) 
In order to capture fast dynamics and large areas, fast scanning techniques are applied. One of the most interesting techniques operates by employing AOD pairs [56]. By propagating sound waves through an optical crystal, AOD works as a tunable light deflector, and by changing the frequency of the acoustic waves, it steers the laser beam in one dimension. By orthogonally stacking multiple AODs, it can provide 3-D scanning abilities [57]. Comparing with mechanical scanning components such as galvo mirrors, AOD scanner have a higher operating frequency, and can provide precise access to the spot of interest. As shown in Figure 16, by employing the state-of-the-art technology, multiscale automated scanning microscopy can be realized in a large area for several days of continuous observation.Research have been done in disease models using two photon based calcium imaging, including work on Alzheimer's disease (AD), stroke, ischemia, hypoxia and epilepsy [58]. Although the cause and progression of $\mathrm{AD}$ remains not well understood, it is believed that the senile plaques and neurofibrillary tangles which accumulate are associated with the disease [59]. The beta amyloid (A $\beta$ ) can be fluorescently labelled in vivo, and by imaging $\mathrm{A} \beta$ and the neurons simultaneously, it is possible to study the toxicity of $\mathrm{A} \beta$ plaque and the evolution of neuronal damage [60] Research has shown that glial cells are also strongly involved in the development of AD. In vivo imaging of calcium signaling has shown abnormal astrocyte activity and instability at the early stage of the disease, before the onset of $A \beta$ plaque deposition [60].

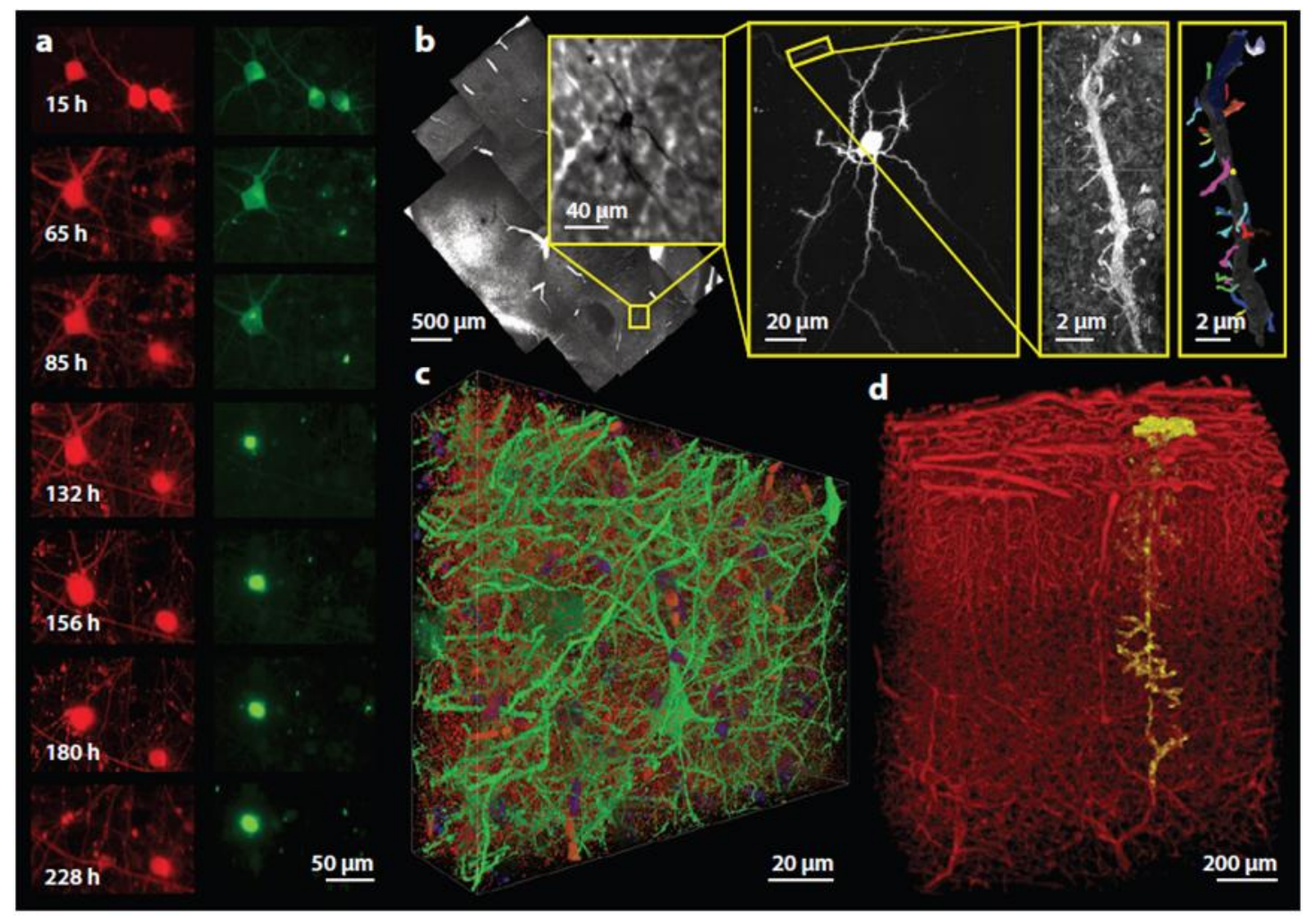

Figure 16:-Automated and multiscale fluorescent microscopy [61] (a) Automated microscopy. (b). Multiscale microscopy. (c). Array tomography. (d). All-optical histology.

\section{Non-invasive Functional Imaging:-}

Hemoglobin is the most significant contributor to light absorption in brain. Since oxy- and deoxyhemoglobin $\left(\mathrm{HbO}_{2}\right.$ and $\mathrm{HbR}$ ) possess unique and distinctive absorption spectra at visible and near infrared wavelength (Figure 16), vascular dynamics can be measured by spectroscopy. Functional activities in brain are closely correlated with cerebral blood flow, blood volume and oxygenation. As a result, brain function can be captured by analyzing the spectra of hemodynamic response.

Infrared light is preferable for spectroscopy because of its low scattering rate in the tissue. And to determine $\mathrm{HbO}_{2}$, $\mathrm{HbR}$ and $\mathrm{Hb}$ total $(\mathrm{HbT})$ signals respectively, two or more wavelengths must be used simultaneously. Calculation of $\mathrm{HbO}_{2}, \mathrm{HbR}$ and $\mathrm{HbT}$ concentration can be done by applying the Beer-Lambert law, in which the effect of light scattering must be taken into consideration [62]. Because of the scattering, photons have traveled different distances 
before collected by the detector. Most photons traveled through very superficial layers of the cortex, while few of them has penetrated deeply into the brain tissue and returned. The differential path length factor (DPF) is used to describe this differential attenuation effect. Light with different wavelengths possess different DPF, and it should impact the accuracy of the result when multiple wavelengths are used.

Comparing with other functional imaging modalities, fNIRS has demonstrated unique utility. EEG has high temporal resolution, but it is difficult to determine the spatial location of the signal source. PET and fMRI provides excellent spatial resolution, but the data acquisition time is long. FNIRS provides moderate spatial and temporal resolution. It can acquire data rapidly with hundreds of Hertz frequency, and generate three dimensional spatial pictures combined with tomosynthesis, although without the ability to identify anatomical structure. For infants, PET and fMRI are not well suited because of the radiation risk and artifact cause by patient movement. Infrared light can penetrate through the skin and skull of a baby much easier than an adult, which also makes fNIRS an ideal method for functional brain imaging of awake infants demonstrated in Figure 17. In addition, fNIRS is able to record brain reactions to auditory stimuli, which is impossible in fMRI.
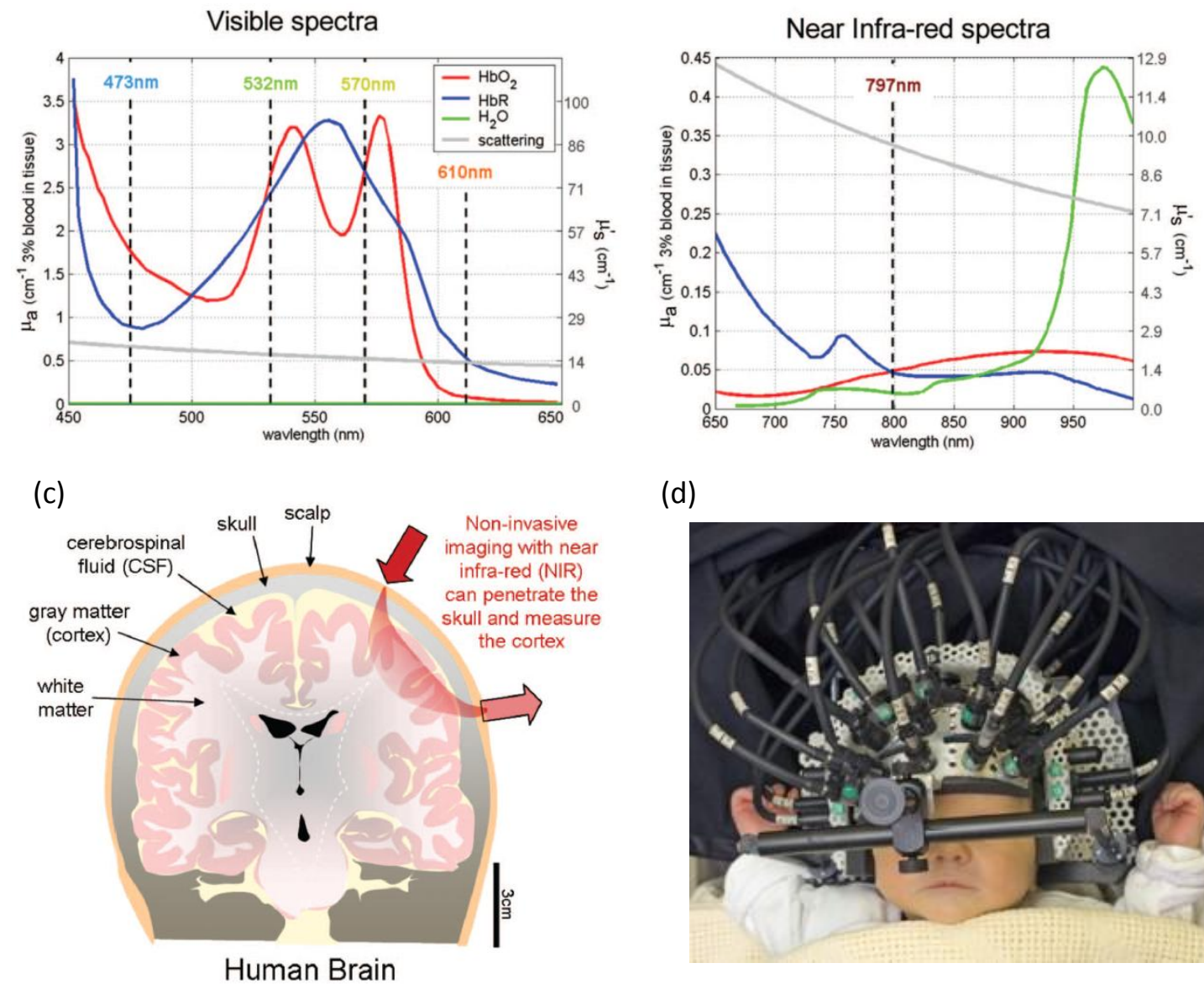

(d)

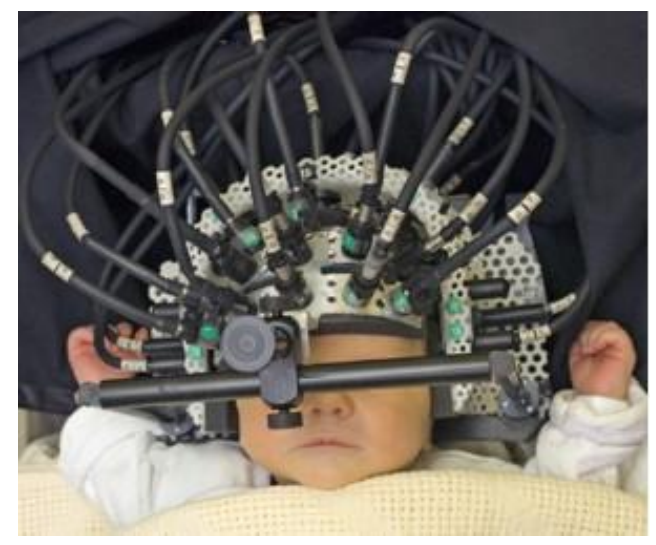

Figure 17:- Absorption spectra of $\mathrm{HbO} 2, \mathrm{HbR}$ and $\mathrm{HbT}$ at (a) visible and (b) near infrared wavelength. Light scattering and water absorption curves are also shown. (c). Schematic of non-invasive fNIRS functional brain imaging and its application on an infant (d) [63]

Although both BOLD fMRI and fNIRS are designed to detect hemodynamic signals, researches have revealed certain discrepancies. One most debated phenomenon is the "initial dip" [64]. Local HbR concentration increases in a short time after stimulation, before it enters a substantial decrease. One possible explanation for this transient change is that local $\mathrm{HbO}_{2}$ is converted to $\mathrm{HbR}$ quickly after activation, before it is replenished from blood flow. However, this dip is only seen in a few BOLD fMRI and many optical studies [65], which suggest that it may be artifact related to the specific experimental conditions such as object species, stimuli type, and measurement parameters. 


\section{Co-Registered fMRI and fNIRS:-}

Although both fMRI and fNIRS target the hemodynamic response, they are based on different physics principles and possess unique advantages. Because fNIRS cannot provide anatomical references, co-registering with MRI is required for signal source localization.

Figure 18 shows an example of a 3-D functional fNIRS mapping fused with MRI image. It is also suggested that fNIRS is best correlated with fMRI signals [42]. BOLD fMRI signal reflects dynamic changes in deoxy-hemoglobin concentrations. However, this change may result from either an increase in oxygenation or a decrease in blood volume, which could be caused by various physiological conditions. By correlating fNIRS results with fMRI, the most accurate data interpretations can be performed. In this work, we reviewed the principles of functional brain imaging for photon microscopy and near infrared spectroscopy. Optical imaging technology has gone through dramatic improvements in recent years. Multiphoton fluorescent microscopy in vivo has led to many ground breaking understanding of neuronal activity, while fNIRS has led to ground breaking clinical research on infants. Future developments in instrumentation, data analysis and multimodality methods should further advance functional optical imaging, and provide promising research tools for both laboratory and clinical applications.

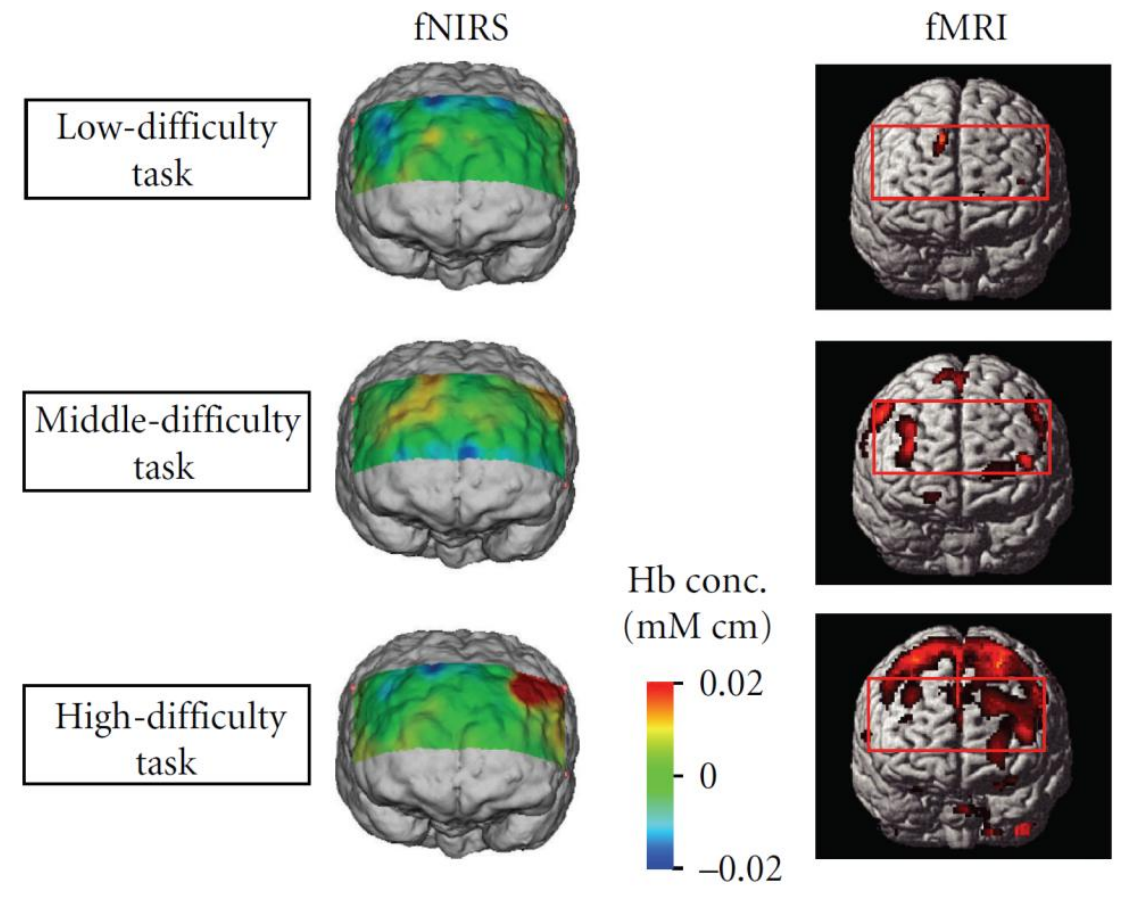

Figure 18:-Co-registered functional brain imaging by fMRI and fNIRS, showing good signal correlation [66]

\section{Conclusion:-}

Functional brain imaging and cognitive neuroscience have been broadly interests of researchers. Various scientistsfrom different departments such as computer science and electrical engineering have demonstrated their motivation to develop methods, algorithms and to design hardware for this branch of brain imaging. This review paper designed for engineers and physical scientists aimed to familiarize audiences with the fundamental of various modalities in functional brain imaging - especially functional MRI. This work also attempted to explore the relationship between engineering and physical aspects of modalities and the physiological and functional activities in the brain. Using the information provided in such review papers enables researchers to open new avenues of transferring neuroimaging challenges from pure research to clinical applications and also improving the quality of data processing and analyses.

\section{Disclosure statement:-}

The authors declare that there is no conflict of interest regarding the publication of this manuscript. 


\section{References:-}

1. C. Goetz, Textbook of Clinical Neurology, 3rd Edition, Saunders,, 2007.

2. Donald C. Johanson, Blake Edgar, From Lucy To Language, Simon and Schuster, 2006.

3. W. Toga, "Neurovascular coupling and imaging techniques," 15 April 2012. [Online]. Available: http://www.loni.ucla.edu/SVG/index.php?vid=261.

4. M. G.-G. Giorgio Carmignoto, "The contribution of astrocyte signalling to neurovascular coupling," BRAIN RESEARCH REVIEWS 63, p. 138 - 148, 2010.

5. J. Ward, The Student's Guide to Cognitive Neuroscience, Psychology Press, 2006.

6. Richard D. Lane, "The Rebirth of Neuroscience in Psychosomatic Medicine, Part I: Historical Context, Methods, and Relevant Basic Science," Psychosomatic Medicine 71, p. 117-134, 2009.

7. Ernest Niedermever, Fernando Lopes da Silva, Electroencephalography: Basic Principles, Clinical Applications, and Related Fields, Lippincott Williams \& Wilkins, 2004.

8. Michael S. Gazzaniga, R. Ivry, G. R. Mangun, Fundamentals of cognitive neuroscience, New York: Norton, 1998.

9. M. Teplan, "Fundamentals of EEG measurement," Measurement science review, pp. 1-11, 2002.

10. Mohammad A. Rahman, Wanli Ma, Dat Tran, John Campbell, "A comprehensive survey of the feature extraction methods in the EEG research," Algorithms and Architectures for Parallel Processing, pp. 274-283, 2012.

11. Sean Jenkins, Raymond Brown, and Neil Rutterford, "Comparing thermographic, EEG, and subjective measures of affective experience during simulated product interactions.," International journal of Design, 2009.

12. Matti Hämäläinen , Riitta Hari, Risto J. Ilmoniemi, Jukka Knuutila, and Olli V. Lounasmaa. , "Magnetoencephalography - theory, instrumentation, and applications to noninvasive studies of the working human brain.," Reviews of modern Physics, 1993.

13. L. Lauronena, "Magnetoencephalography in neonatology," Clinical Neurophysiology, pp. 27-34, 2011.

14. Vittorio Pizzella, Laura Marzetti, Stefania Della Penna, Francesco de Pasquale, Filippo Zappasodi, and Gian Luca Romani, "Magnetoencephalography in the study of brain dynamics," Functional neurology, 2014.

15. S. P. Singh, "Magnetoencephalography: Basic principles.," Annals of Indian Academy of Neurology, 2014.

16. M. M. Alauddin, "Positron emission tomography (PET) imaging with 18F-based radiotracers.," Am J Nucl Med Mol Imaging, pp. 55-76, 2012.

17. Peter E. Valk, Positron Emission Tomography Clinical Practice, Springer, 2006.

18. U. Kiendys, "F-18 fl uorodeoxyglucose (FDG) positron emission tomography (PET)-positive parotid incidentaloma: Prevalence and clinical signifi cance," Reports in Medical Imaging, p. 1-6, 2009.

19. Y.F. Tai, P. Piccini, "Applications of positron emission tomography (PET) in neurology," Journal of Neurology, Neurosurgery \& Psychiatry, 75(5), pp.., pp. 669-676, 2004.

20. G. N. Morano, "Technical Overview of Brain SPECT Imaging," JOURNAL OF NUCLEAR MEDICINE TECHNOLOGY, pp. 191-195, December 2003.

21. C. Harrison, "Single photon emission computed tomography and its use in neuroimaging," Clinical Eye and Vision Care, pp. 139-147, 1999.

22. S. A. Huettel, Functional Magnetic Resonance Imaging, Sunderland, Massachuesetts, USA: Sinauer Associates, Inc, 2004.

A. W. Toga et al., Brain Mapping The Methods, Academic Press, 2002.

23. W. Toga et al., "Neurovascular coupling and imaging techniques," 15 April 2012. [Online]. Available: http://www.loni.ucla.edu/SVG/index.php?vid=261.

24. J.B.M. Warntjes, CEO, SyntheticMR AB, "Magnetic Resonance Imaging of the future will show brain structures automatically in PACS," 23 October 2009. [Online]. Available: http://newsroom.sectra.com/2009/10/magnetic-resonance-imaging-of-the-future-will-show-brain-structuresautomatically-in-pacs/. [Accessed 19 April 2012].

25. S.-G. Kim, "Functional Magnetic Resonance Imaging of Human Brain," Neuroscience Methods, pp. 229-243, 1997.

26. S.M. Smith, M. Jenkinson, M. Woolrich, C. Beckmann, T. Behrens, H. Johansen-Berg, P. Bannister, M. De Luca, I. Drobnjak, D. Flitney, R. Niazy, J. Saunders, J. Vickers, Y. Zhang, N. De Stefano, J. Brady, P. Matthews, "Advances in functional and structural MR image analysis and implementation as FSL," NeuroImage,, vol. 23, pp. 208-19, 2004.

27. Stephen C. Strother, Saman Sarraf, Cheryl Grady, "A Hierarchy of Cognitive Brain Networks Revealed by Multivariate Performance Metrics," in Signals, Systems and Computers, 2014 48th Asilomar Conference, Asilomar, 2014. 
28. P. Bellec, "Multi-level bootstrap analysis of stable clusters in resting-state fMRI," NeuroImage 51, p. 11261139, 2010.

29. Y. Liu et al., "Regional homogeneity, functional connectivity and imaging markers of Alzheimer's disease: A review of resting-state fMRI studies," Neuropsychologia 46, p. 1648-1656, 2008.

30. M. P. v. d. Heuvel, "Exploring the brain network: A review on resting-state fMRI functional connectivity," European Neuropsychopharmacology, p. 519-534, 2010.

31. SM Hadi Hosseini, Fumiko Hoeft, and Shelli R. Kesler, "GAT: a graph-theoretical analysis toolbox for analyzing between-group differences in large-scale structural and functional brain networks," PloS one, 2012.

32. Cheryl Grady, Saman Sarraf, Cristina Saverino, and Karen Campbell, "Age differences in the functional interactions among the default, frontoparietal control, and dorsal attention networks.," Neurobiology of aging, pp. 159-172, 2016.

33. Saman Sarraf, Cristina Saverino, and Ali Mohammad Golestani, "A robust and adaptive decision-making algorithm for detecting brain networks using functional mri within the spatial and frequency domain," in In 2016 IEEE-EMBS International Conference on Biomedical and Health Informatics (BHI), Las Vegas, NV, 2016.

34. Cristina Saverino, Zainab Fatima, Saman Sarraf, Anita Oder, Stephen C. Strother, and Cheryl L. Grady, "The Associative Memory Deficit in Aging Is Related to Reduced Selectivity of Brain Activity during Encoding.," Journal of cognitive neuroscience, 2016.

35. David W. Townsend, "Dual-Modality Imaging: Combining Anatomy and Function," THE JOURNAL OF NUCLEAR MEDICINE Vol. 49 • No. 6, pp. 938-955, 2008.

36. Arno Villringer, Christoph Mulert, and Louis Lemieux, EEG-fMRI, Verlag Berlin Heidelberg: Springer, 2010.

37. F. F. Jobsis et al., "Noninvasive, infrared monitoring of cerebral and myocardial oxygen sufficiency and circulatory parameters," Science 198, p. 1264, 1977.

38. R. Y. Tsien et al., "A non-disruptive technique for loading calcium buffers and indicators into cells," Nature 290 , p. $527,1981$.

39. W. Denk et al., J. H. Strickler, and W. W. Webb, “, "Two-photon laser scanning fluorescence microscopy," Science 248, p. 73, 1990.

40. P. M. Arentha, J. H. Ricker and M. T. Schultheis, "Applications of Functional Near-Infrared Spectroscopy (fNIRS) to Neurorehabilitation of Cognitive Disabilities," The Clinical Neuropsychologist 21, p. 38, 2007.

41. V. Q. M. Ferrari et al., "A brief review on the history of human functional near-infrared spectroscopy (fNIRS) development and fields of application," Brain and Language 121, p. 79 , 2012.

42. Hock, K. Villringer, F. Mullerspahn, M. Hofmann, S. Schuhhofer, H. Heekeren, R. Wenzel, U. Dirnagl, A. Villringer, "Near Infrared Spectroscopy in the Diagnosis of Alzheimer's Disease," Annals of the New York Academy of Sciences 777, p. 22, 1996.

43. F. Irania, S. M. Platek, S. Bunce, A. C. Ruocco and D. Chute, "Functional Near Infrared Spectroscopy (fNIRS): An Emerging Neuroimaging Technology with Important Applications for the Study of Brain Disorders," The Clinical Neuropsychologist 21, p. 9, 2007.

44. G. Strangman, R. Goldstein, S. L. Rauch, and J. Stein, "Nearinfrared spectroscopy and imaging for investigating stroke rehabilitation: test-retest reliability and review of the literature," Arch. Phys. Med. Rehabil. 87, p. 12, 2006.

45. L. A. Nelson, J. C. McCann, A. W. Loepke, J. Wu, B. B. Dor, and C. D. Kurth, "Development and validation of a multiwavelength spatial domain near-infrared oximeter to detect cerebral hypoxiaischemia," J. Biomed. Opt. 11, 064022, 2006.

46. J. S. Wyatt, A. D. Edwards, D. Azzopardi, C. E. Richardson, M. Cope, D. T. Delpy, E. B. Cady and E. O. R. Reynolds, "Cerebral Heamodynamics During Failure of Oxidative Phosphorylation Following Birth Asphyxia," Pediatric Research 26, p. 511, 1989.

47. F. van Bel, C. A. Dorrepaal, M. J.N.L. Benders, P. E.M. Zeeuwe, M. van de Bor, and H. M. Bergen, "Changes in Cerebral Hemodynamics and Oxygenation in the First 24 Hours After Birth Asphyxia," Pediatrics 92, p. 365, 1993.

48. M. C. Toet, P. M. A. Lemmers, "Brain monitoring in neonates," Early Human Development 85, p. 77, 2009.

49. P. F. Moulton et al., "Spectroscopic and laser characteristics of Ti:Al2O3," J. Opt. Soc. B 3, p. 125 , 1986.

50. K. Ohki, S. Chung, P. Kara, M. Hubener, T. Bonhoeffer, and R. C. Reid, "Highly ordered arrangement of single neurons in orientation pinwheels," Nature 24, p. 925, 2006.

51. M. Zonta, M. C. Angulo, S. Gobbo, B. Rosengarten, K. A. Hossmann, T. Pozzan, and G. Carmignoto, "Neuronto-astrocyte signaling is central to the dynamic control of brain microcirculation," Nat. Neurosci. 6, p. 43, 2003. 
52. T. Takano, G. Tian, W. Peng, N. Lou, W. Libionka, X. Han, and M. Nedergaard, "Astrocyte-mediated control of cerebral blood flow," Nat. Neurosci. 9, p. 260, 2006.

53. Brian A.Wilt et al., "Advances in Light Microscopy," Annu.Rev.Neurosci. 32,, p. 435, 2009.

54. R. Cossart, Y. Ikegaya and R. Yuste, "Calcium imaging of cortical networks dynamics," Cell Calcium 37, p. 451, 2005.

55. Bullen, S. S. Patel, and P. Saggau, "High-speed, random-access fluorescence microscopy: I. High-resolution optical recording with voltage-sensitive dyes and ion indicators," Biophys. J. 73, p. 477, 1997.

56. G. D. Reddy, K. Kelleher, R. Fink, and P. Saggau, "Three-dimensional random access multiphoton microscopy for functional imaging of neuronal activity," Nat. Neurosci. 11, p. 713, 2008.

57. N. L. Rochefort, H. Jia and A. Konnerth, "Calcium imaging in the living brain: prospects for molecular medicine," Trends in Molecular Medicine 14, p. 389, 2008.

58. P. Tiraboschi, L. A. Hansen, L. J. Thal, and J. Corey-Bloom, "The importance of neuritic plaques and tangles to the development and evolution of AD"," Neurology 62, p. 1984, 2004.

59. M. Meyer-Luehmann, T. L. Spires-Jones, C. Prada, M. Garcia-Alloza, A. de Calignon, A. Rozkalne1, J. Koenigsknecht-Talboo, D. M. Holtzman, B. J. Bacskai and B. T. Hyman,, "Rapid appearance and local toxicity of amyloid- plaques in a mouse model of Alzheimer's disease," Nature 451, p. 720, 2008.

60. A.Wilt, L. D. Burns, E. T. W. Ho, K. K. Ghosh, E. A. Mukamel, and M. J. Schnitzer, "Advances in light microscopy for neuroscience," Annu. Rev. Neurosci. 32, p. 435, 2009.

61. M. Kohl, U. Lindauer, G. Royl, M. Kuhl, L. Gold, A. Villringer, and U. Dirnagl, "Physical model for the spectroscopic analysis of cortical intrinsic optical signals," Phys. Med. Biol. 45, p. 3749, 2000.

62. E. M. C. Hillman., "Optical brain imaging in vivo: techniques and applications from animal to man," J. Biomed. Opt. 12, 051402, 2007.

63. Malonek , and A. Grinvald,, "Interactions between electrical activity and cortical microulation revealed by imaging spectroscopy: implications for functional brain mapping," Science 272, p. 551, 1996.

64. R. S. Menon, S. Ogawa, X. Hu, J. P. Strupp, P. Anderson, and K. Ugurbil, "BOLD based functional MRI at 4 Tesla includes a capillary bed contribution: echo-planar imaging correlates with previous optical imaging using intrinsic signals," Nat. Neurosci. 33, p. 453, 1995.

65. Hitoshi Tsunashima, Kazuki Yanagisawa, "Measurement of brain function of car driver using functional nearInfrared spectroscopy (fNIRS)," Computational intelligence and neuroscience, 2009.

66. B. E. Donald C. Johanson et al., From Lucy To Language, Simon and Schuster, 2006.

67. Sarraf, S. H., Marzbanrad, E., Mobedi, H., Rafienia, M., Mirzadeh, H., Jamshidi, A., "Simulation Of Betamethasone Release Profiles From In Situ Forming Systems Based On Plga," in Canadian Medical and Biological Engineering Society (CMBEC32), Calgary, Alberta, 2009.

68. Saman Hossein Sarraf, Ehsan Marzbanrad, Hamid Mobedi, Mohammad Rafienia,Hamid Mirzadeh,Ahmad Jamshidi, "SIMULATION OF BETAMETHASONE RELEASE PROFILES FROM IN SITU FORMING SYSTEMS BASED ON PLGA," in 32nd Conference of the Canadian Medical and Biological Engineering Society (CMBEC32), Calgary, Alberta, 2009.

69. Saman Sarraf, Cristina Saverino, Halleh Ghaderi, John Anderson, "Brain network extraction from probabilistic ICA using functional Magnetic Resonance Images and advanced template matching techniques," in Electrical and Computer Engineering (CCECE), 2014 IEEE 27th Canadian Conference, Toronto, 2014.

70. Saman Sarraf, Raghda Hasswa, Carol Dematteo, John Connolly, and Michael D. Noseworthy, "Resting-State fMRI Data Classification of Exercise-Induced Brain Changes in Healthy Subjects Using Probabilistic Independent Component Analysis (PICA)," Researchgate, 2013.

71. Saman Sarraf, Ehsan Marzbanrad, Hamid Mobedi, "Mathematical Modeling for Predicting Betamethasone Profile and Burst Release From In Situ Forming Systems Based On PLGA," in Electrical and Computer Engineering (CCECE), 2014 IEEE 27th Canadian Conference on, Toronto, 2014.

72. M. A. Warsi, "The Fractal Nature and Functional Connectivity of Brain Function as Measured by BOLD MRI in Alzheimer's Disease," macsphere.mcmaster.ca, Hamilton, 2012.

73. David J. Heeger, David Ress, "What does fMRI tell us about neuronal activity?," Nature Reviews Neuroscience, pp. 142-151, 2002.

74. O. Garaschuk, R.I. Milos, and A. Konnerth, "Targeted bulk-loading of fluorescent indicators for two-photon brain imaging in vivo," Nat Prot 1, p. 380, 2006. 